Pacific

Journal of

Mathematics

THE MACROSCOPIC SOUND OF TORI

Constantin Vernicos

Volume 213 No. 1

January 2004 


\title{
THE MACROSCOPIC SOUND OF TORI
}

\author{
Constantin Vernicos
}

\begin{abstract}
Take a torus with a Riemannian metric. Lift the metric on its universal cover. You get a distance which in turn yields balls. On these balls you can look at the Laplacian. Focus on the spectrum for the Dirichlet or Neumann problem. We describe the asymptotic behaviour of the eigenvalues as the radius of the balls goes to infinity, and characterise the flat tori using the tools of homogenisation our conclusion being that "Macroscopically, one can hear the shape of a flat torus". We also show how in the two dimensional case we can recover earlier results by D. Burago, S. Ivanov and I. Babenko on the asymptotic volume.
\end{abstract}

\section{Introduction and claims.}

Let $\left(\mathbb{T}^{n}, g\right)$ be a Riemannian torus, lift its metric on its universal cover and use it to define first a distance, then the metric's balls. The first thing one can observe is the volume of these balls as a function of their radius, indeed as the distance obtained arises from a compact quotient it is equivalent to an Euclidean distance hence the volume of these balls is equivalent to the Euclidean volume of an Euclidean ball i.e., proportional to the radius of the ball to the power of $n$ (the dimension of our torus).

We are thus naturally led to wonder what happens if one looks at the following Riemanniann function on the balls $\left(B_{g}(\rho)\right.$ is the ball of radius $\left.\rho\right)$ :

$$
\frac{\operatorname{Vol}_{g}\left(B_{g}(\rho)\right)}{\rho^{n}} \text { as } \rho \rightarrow+\infty \text {. }
$$

If it is not very surprising that it converges to some constant for this limit can be seen as a mean value due to the periodicity of the metric (see for example Pansu [Pan82] and a slightly different and more analytical proof in this paper Section 2.3), it is quite remarkable that this constant, called asymptotic volume, is bounded from below by the constant arising from the flat tori and furthermore that the case of equality caracterises the flat tori as D. Burago and S. Ivanov showed in [BI95].

The study of the balls of large radii on the universal cover of tori (and more generally of a nilmanifold) is what we call here the macroscopical geometry. Indeed in our case the universal cover is a real vector space, where 
some lattices acts by translation (in the more general case of nilmanifolds one should consider a left action). Should one focus on the point of this lattice endowed with the distance arising from the torus, one gets an invariant metric on the lattice. Now if one looks at this lattice from a galaxy far, far away, one won't be able to distinguish the lattice from the whole universal cover. Thus it is understandable that for this observer the distance obtained on the universal cover seems invariant by all translations (for general nilmanifolds one gets a left invariant distance).

In the case of tori this "seen from a far away galaxy" distance is a norm, called the stable norm and was first defined by Federer in homology. It is some kind of mean value of the metric. This asymptotic behaviour was generalized and proved by P. Pansu for all nilmanifold [Pan82] and precised by D. Burago [Bur92] for tori. Since then the stable normed appeared in many other works: For surfaces and the links with Aubry-Mather theory in D. Massart's works, one can also find it in the weak KAM theory of A. Fathi. It is also worth mentionning the crucial role it plays in the proof by D. Burago and S. Ivanov [BI94] of the Hopf conjecture concerning tori without conjugate points. Here in Part 2 we show, for the case of tori, how one recovers the stable norm using homogenisation tools.

There is another interesting geometric invariant attached to the balls and linked with the volume, the spectrum of the Laplacian. Indeed if one knows the spectrum one knows the volume thanks to Weyl's asymptotic formula. Here again one easily sees, comparing with the Euclidean case, that the eigenvalues converge to zero with a $1 / \rho^{2}$ speed ( $\rho$ being the radius). If one can expect a convergence when rescaled, it is quite surprising that as a limit we obtain the spectrum of an Euclidean and not a finsler metric, indeed the behaviour is described by the following theorem which is one of the aims of this paper:

Theorem 1. Let $\left(\mathbb{T}^{n}, g\right)$ be a Riemannian torus, $B_{g}(\rho)$ the induced metric ball on its universal cover and $\lambda_{i}\left(B_{g}(\rho)\right)$ the $i^{\text {th }}$ eigenvalue of the Laplacian for the Dirichlet (resp. Neumann) problem.

There exists an elliptic operator $\Delta_{\infty}$, which is the Laplacian of some Euclidean metric on $\mathbb{R}^{n}$, such that if $\lambda_{i}^{\infty}$ is its $i^{\text {th }}$ eigenvalue for the Dirichlet (resp. Neumann) problem on the stable's norm unit ball then

$$
\lim _{\rho \rightarrow+\infty} \rho^{2} \lambda_{i}\left(B_{g}(\rho)\right)=\lambda_{i}^{\infty} .
$$

Section 4 is devoted to the proof of this theorem and Section 3 introduces the analytical background: Homogenisation and various convergence fairly known by the specialist of homogenisation but adapted here to our purpose, as it becomes after some re-spelling of the problem made in Section 2.4. From this theorem we can deduce the following one which is some kind of "à la" Burago-Ivanov macroscopical rigidity and which inspired the abstract: 
Theorem 2. Let $\left(\mathbb{T}^{n}, g\right)$ be a Riemannian torus, $B_{g}(\rho)$ the induced metric ball on its universal cover and $\lambda_{1}\left(B_{g}(\rho)\right)$ the first eigenvalue of the Laplacian for the Dirichlet problem. Then:

(1) $\lim _{\rho \rightarrow+\infty} \rho^{2} \lambda_{1}\left(B_{g}(\rho)\right)=\lambda_{1}^{\infty} \leq \lambda_{e, n}$,

(2) equality holds if, and only if, the torus is flat, where $\lambda_{e, n}$ is the first eigenvalue of the Euclidean Laplacian on the Euclidean unit ball.

The proof, which is done in Section 6, involves some kind of transplantation for the inequality mixed with $\Gamma$-convergence for the equality. For a better understanding of what happens we briefly give some informations related to the $\Gamma$-convergence and adapt it to our purpose in Section 5 , following the general ideas of K. Kuwae and T. Shioya in [KS] (who in turn generalized U. Mosco's paper [Mos94]), this section being completed by the proof of Section 8.

As the macroscopical spectrum involved rises from an Euclidean metric, we can use the Faber-Krahn inequality to obtain a new inequality regarding the asymptotic volume, this is done in Section 7.1:

Proposition 3. Let $\left(\mathbb{T}^{n}, g\right)$ be a Riemannian torus, $B_{g}(\rho)$ the geodesic balls of radius $\rho$ centred on a fixed point and $\operatorname{Vol}_{g}\left(B_{g}(\rho)\right)$ their Riemannian volume induced on the universal cover, writing

$$
\operatorname{Asvol}(g)=\lim _{\rho \rightarrow \infty} \frac{\operatorname{Vol}_{g}\left(B_{g}(\rho)\right)}{\rho^{n}}
$$

then:

(1) $\operatorname{Asvol}(g) \geq \frac{\operatorname{Vol}_{g}\left(\mathbb{T}^{n}\right)}{\operatorname{Vol}_{\mathrm{Al}}\left(\mathbb{T}^{n}\right)} \omega_{n}$.

(2) In case of equality, the torus is flat.

Here $\omega_{n}$ is the unit Euclidean ball's Euclidean volume, and $\operatorname{Vol}_{\mathrm{Al}}\left(\mathbb{T}^{n}\right)$ is the volume of the Albanese torus.

A surprising fact arises because this new inequality involves a constant which happened to be at the heart of the isosystolic inequality of two dimensional tori (see J. Lafontaine [Laf74]), hence we obtain an alternate proof of the asymptotic volume's lower boundedness in dimension two:

Corollary 4. Let $\left(\mathbb{T}^{2}, g\right)$ be a 2-dimensional torus then:

(1) $\operatorname{Asvol}(g) \geq \pi$.

(2) In case of equality, the torus is flat.

It is worth mentionning that the case of equality in the previous claims relies on Theorem 33, which states that the stable norm coincides with the Albanese metric if and only if the torus is flat, and whose proof does not rely on the work of D. Burago and S. Ivanov [BI95] or I. Babenko [Bab91]. 
Thus we actually get an alternate proof of this theorem in the 2-dimensional case.

We also give a kind of generalised Faber-Krahn inequality for normed finite dimensional vector spaces, which implies that we cannot distinguish the Euclidean's ones among them using the first generalised eigenvalue of the Dirichlet Laplacian (see Lemma 36 and its corollary):

Theorem 5 (Faber-Krahn inequality for norms). Let $D$ be a domain of $\mathbb{R}^{n}$, with the norm $\|\cdot\|$ and a measure $\mu$ invariant by translation. Let $D^{*}$ be the norm's ball with same measure as $D$, then

$$
\lambda_{1}\left(D^{*},\|\cdot\|\right) \leq \lambda_{1}(D,\|\cdot\|) .
$$

We finally explain in Section 7.2 how is our work related to works focused on the long time asymptotics of the heat kernel (see [KS00], [DZ00], [ZKON79]) and finally in Section 7.3 we state how Theorem 1 transposes to all graded nilmanifolds (subject which should be widely extended in a forthcoming article).

\section{Stable norm and homogenisation.}

In this section we show how the stable norm, the Gromov-Hausdorff convergence and the $\Gamma$-convergence of the homogenisation theory are linked and finish by re-spelling our goal. In what follows, $B_{g}(\rho)$ will be the metric ball of radius $\rho$ on the universal cover of a torus with the lifted metric. We first begin by two definitions.

2.1. Convergences. We recall the definition of $\Gamma$-convergence in a metric space:

Definition 6. Let $(X, d)$ be a metrics space. We say that a sequence of function $\left(F_{j}\right)$ from $X$ to $\overline{\mathbb{R}}, \Gamma$-converges to a function $F: X \rightarrow \overline{\mathbb{R}}$ if and only if for all $x \in X$ we have:

(1) For all converging sequences $\left(x_{j}\right)$ to $x$

$$
F(x) \leq \liminf _{j \rightarrow \infty} F_{j}\left(x_{j}\right)
$$

(2) there exists a sequence $\left(x_{j}\right)$ converging to $x$ such that

$$
F(x)=\lim _{j \rightarrow \infty} F_{j}\left(x_{j}\right) .
$$

We now introduce the Gromov-Hausdorff measured convergence in the space $\mathcal{M}$ of compact metric and measured spaces $(X, d, m)$ modulo isometries. First if $X$ and $Y$ are in $\mathcal{M}$ then an application $\phi: X \rightarrow Y$ is called an $\epsilon$-Hausdorff approximation if and only if we have:

(1) The $\epsilon$-neighbourhood of $\phi(X)$ in $Y$ is $Y$; 
(2) for all $x, y \in X$ we have

$$
|d(x, y)-d(\phi(x), \phi(y))| \leq \epsilon .
$$

We write $C^{0}(X)$ for the space of continues functions from $X$ to $\mathbb{R}$ and $\mathcal{A}$ will be a partially directed space.

Definition 7. We say that a net $\left(X_{\alpha}, d_{\alpha}, m_{\alpha}\right)_{\alpha \in \mathcal{A}}$ of spaces in $\mathcal{M}$ converges to $(X, d, m)$ for the Gromov-Hausdorff measured topology if, and only if there exists a net of positive real numbers $\left(\varepsilon_{\alpha}\right)_{\alpha \in \mathcal{A}}$ decreasing to 0 and $m_{\alpha}$ measurable $\varepsilon_{\alpha}$-Hausdorff approximations $f_{\alpha}: X_{\alpha} \rightarrow X$ such that $\left(f_{\alpha}\right)_{*}\left(m_{\alpha}\right)$ converges vaguely to $m$ i.e.,

$$
\int_{X_{\alpha}} u \circ f_{\alpha} d m_{\alpha} \rightarrow \int_{X} u d m \quad \forall u \in C^{0}(X) .
$$

2.2. The stable norm. Let $\left(\mathbb{T}^{n}, g\right)$ a Riemannian torus. We will call rescaled metrics the metrics $g_{\rho}=\left(1 / \rho^{2}\right)\left(\delta_{\rho}\right)^{*} g$ and their lifts on the universal cover. We will also write $\delta_{\rho}$ for the homothetie of scale $\rho$.

In the 80's P. Pansu showed that the distance induced on $\mathbb{R}^{n}$ as a universal cover of a torus, behaved asymptotically like the distance induced by a norm. In the 90's D. Burago showed a similar result for periodic metrics on $\mathbb{R}^{n}$. It is that norm which is called the stable norm. To be more precise let us write $f_{1}(x)=d_{g}(0, x)$ the distance from the origin to $x$ and $f_{\rho}(x)=d_{g}\left(0, \delta_{\rho}(x)\right) / \rho$, then P. Pansu's result says that there exist a norm $\|.\|_{\infty}$ such that for all $x \in \mathbb{R}^{n}$

$$
\lim _{\rho \rightarrow \infty} f_{\rho}(x)=\|x\|_{\infty}
$$

and Burago's says that there exists a constant $C$ such that for all $x \in \mathbb{R}^{n}$

$$
\left|f_{\rho}(x)-\|x\|_{\infty}\right| \leq \frac{C}{\rho}
$$

in other words, Pansu's results is a simple convergence and Burago's is a uniform convergence result.

There is another proof of the simple convergence of the sequence $\left(f_{\rho}\right)$ as $\rho$ goes to infinity, using homogenisation tools.

Theorem 8. Let $\widetilde{g}$ the induced metric on $\mathbb{R}^{n}$ as a universal cover of a Riemannian torus $\left(\mathbb{T}^{n}, g\right)$. Then there exists a norm $\|.\|_{\infty}$ such that:

(1) For every bounded open $I \subset \mathbb{R}$ the sequence of functionals

$$
E_{\rho}(u)=\int_{I} \widetilde{g}_{\left(\delta_{\rho} u(t)\right)}\left(u^{\prime}(t), u^{\prime}(t)\right) d t
$$

on $W^{1,2}\left(I ; \mathbb{R}^{n}\right)$, $\Gamma$-converge for the $L^{2}$ norm toward the functional

$$
E_{\infty}(u)=\int_{I}\left\|u^{\prime}(t)\right\|_{\infty}^{2} d t
$$


(2) the norm satisfies

(1)

$$
\|\xi\|_{\infty}=\lim _{t \rightarrow+\infty} \inf \left\{\frac{1}{t} \int_{0}^{t} \widetilde{g}_{(u+\xi \tau)}\left(u^{\prime}+\xi, u^{\prime}+\xi\right) d \tau: u \in W_{0}^{1,2}(] 0, t\left[; \mathbb{R}^{n}\right)\right\} .
$$

Furthermore if $f_{\rho}(x)=d_{g}(0, \rho x) / \rho$ then for all $x \in \mathbb{R}^{n}$

$$
\lim _{\rho \rightarrow+\infty} f_{\rho}(x)=\|x\|_{\infty} .
$$

Proof. We use Proposition 16.1, p. 142 of A. Braides and A. Desfranceschi [BD98]. It gives us the $\Gamma$-convergence of the sequence of functional $\left(E_{\rho}\right)$ toward a functional $E_{\infty}$ such that

$$
E_{\infty}(u)=\int_{I} \varphi\left(u^{\prime}(t)\right) d t
$$

with $\varphi$ convex and satisfying the asymptotic formula (1). It remains to show that $\varphi$ is the square of a norm.

Homogeneity: Using the asymptotic formula (1) we easily get $\varphi(0)=0$ and by a change of variables $\varphi(\lambda x)=\lambda^{2} \varphi(x)$.

Separation: Let us point out that:

1) The minimum of the energy of a path between 0 and $t \xi$ in an Euclidean space is attained for the straight line. Thus if we put into the asymptotic formula (1) an Euclidean metric, we get the same metric.

2) Let $g$ and $h$ be two metrics such that for all $s$ and $\xi$

$$
g_{s}(\xi, \xi) \leq h_{s}(\xi, \xi)
$$

then for all $u \in W_{0}^{1,2}(] 0, t\left[; \mathbb{R}^{n}\right)$ we get

$$
\frac{1}{t} \int_{0}^{t} g_{(u+\xi \tau)}\left(u^{\prime}+\xi, u^{\prime}+\xi\right) d \tau \leq \frac{1}{t} \int_{0}^{t} h_{(u+\xi \tau)}\left(u^{\prime}+\xi, u^{\prime}+\xi\right) d \tau
$$

thus taking the infimum for $u$ and taking the limit as $t$ goes to infinity we get

$$
\begin{aligned}
& \lim _{t \rightarrow+\infty} \inf \left\{\frac{1}{t} \int_{0}^{t} g_{(u+\xi \tau)}\left(u^{\prime}+\xi, u^{\prime}+\xi\right) d \tau: u \in W_{0}^{1,2}(] 0, t\left[; \mathbb{R}^{n}\right)\right\} \\
& \leq \lim _{t \rightarrow+\infty} \inf \left\{\frac{1}{t} \int_{0}^{t} h_{(u+\xi \tau)}\left(u^{\prime}+\xi, u^{\prime}+\xi\right) d \tau: u \in W_{0}^{1,2}(] 0, t\left[; \mathbb{R}^{n}\right)\right\} .
\end{aligned}
$$

Now let us also remark that $g$ being periodic, there exists two strictly positive constants $\alpha$ and $\beta$ such that

$$
\alpha|\xi|^{2} \leq g_{s}(\xi, \xi) \leq \beta|\xi|^{2}
$$

now applying the three remarks we get

$$
\alpha|\xi|^{2} \leq \varphi(\xi) \leq \beta|\xi|^{2}
$$

thus $\varphi(\xi)=0$ if and only if $\xi=0$. 
Triangle inequality: First note that

$$
\left\{\xi \in \mathbb{R}^{n} \mid \varphi(\xi) \leq 1\right\}=\left\{\xi \in \mathbb{R}^{n} \mid \sqrt{\varphi(\xi)} \leq 1\right\}=S_{n} .
$$

It follows that if $\sqrt{\varphi}(\xi)=1=\varphi(\xi)$ and $\sqrt{\varphi}(\eta)=1=\varphi(\eta)$ then for all $0 \leq \lambda \leq 1$ by the convexity of $\varphi$

$$
\varphi(\lambda \xi+(1-\lambda) \eta) \leq \lambda \varphi(\xi)+(1-\lambda) \varphi(\eta)=1
$$

SO

$$
\sqrt{\varphi}(\lambda \xi+(1-\lambda) \eta) \leq 1 .
$$

Thus for all non-null $x, y$

$$
\sqrt{\varphi}\left(\lambda \frac{x}{\sqrt{\varphi}(x)}+(1-\lambda) \frac{y}{\sqrt{\varphi}(y)}\right) \leq 1
$$

now taking $\lambda=\sqrt{\varphi}(x) /(\sqrt{\varphi}(x)+\sqrt{\varphi}(y))$ and using $\sqrt{\varphi}$ homogeneity we finally get the triangle inequality and we are able to conclude that $\|\cdot\|_{\infty}=$ $\sqrt{\varphi}(\cdot)$ is a norm.

The final assertion comes from the fact that $\|\xi\|_{\infty}^{2}$ is the limit of the energies' infimum along the paths between 0 and $\xi$ for the rescaled metrics $\left(1 / t^{2}\right)\left(\delta_{t}^{*}\right) g$, which are attained along the geodesics.

This theorem easily induces the following assertion:

Corollary 9. For all $x$ and $y \in \mathbb{R}^{n}$ we have

$$
\lim _{\rho \rightarrow+\infty} \frac{d_{g}(\rho x, \rho y)}{\rho}=\|x-y\|_{\infty} .
$$

From now on we will write $d_{\rho}(x, y)=d_{g}(\rho x, \rho y) / \rho$, and we are now going to see what can be deduced for the balls $B_{g}(\rho)$ in terms of Gromov-Hausdorff convergence.

2.3. Gromov-Hausdorff convergence of metric balls. We will write $\mu_{g}$ (resp. $\mu_{\rho}$ ) the measure induced by $\widetilde{g}$ (resp. $\left.g_{\rho}\right) . \mu_{\infty}$ will be the measure of Lebesgue such that for a fundamental domain $D_{f}$ we have $\mu_{\infty}\left(D_{f}\right)=$ $\mu_{g}\left(D_{f}\right)$. Finally let

$$
B_{\rho}(R)=\left\{x \in \mathbb{R}^{n} \mid d_{\rho}(0, x) \leq R\right\}=\frac{1}{\rho} B_{g}(R \cdot \rho),
$$

and

$$
B_{\infty}(R)=\left\{x \in \mathbb{R}^{n} \mid\|x\|_{\infty} \leq R\right\} .
$$

Theorem 10. The net of measured metric spaces $\left(B_{\rho}(1), d_{\rho}, \mu_{\rho}\right)$ converges in the Gromov-Hausdorff measured topology to $\left(B_{\infty}(1),\|\cdot\|_{\infty}, \mu_{\infty}\right)$ as $\rho$ goes to infinity. 
Proof. Let us choose an $\epsilon>0$. We first show that the identity is an $\epsilon$ approximation if $\rho$ is large enough. It suffice to show that there is a finite family of points $\left(x_{1}, \ldots, x_{N}\right)$ such that its $\epsilon$-neighbourhood in $\left(B_{\infty}(1), d_{\infty}\right)$ and, for $\rho$ large enough, in $\left(B_{\rho}(1), d_{\rho}\right)$ is respectively $B_{\infty}(1)$ and $B_{\rho}(1)$ and such that for all $i, j=1, \ldots, N$ we have

$$
\left|\left\|x_{i}-x_{j}\right\|_{\infty}-d_{\rho}\left(x_{i}, x_{j}\right)\right| \leq \epsilon .
$$

Let $r>0$ and let $\left(\gamma_{1}, \ldots, \gamma_{N}\right)$ be all the images of 0 by the action of $\mathbb{Z}^{n}$, such that for $i=1, \ldots, N, \gamma_{i} \in B_{\infty}(r)$. Then we take for $i=1, \ldots, N$, $x_{i}=\gamma_{i} / r$. Let us remark that for $\rho$ large enough these points will all be in $B_{\rho}(1)$.

Now let us point out that, because of the invariance by the $\mathbb{Z}^{n}$ action, there are two constants $\alpha$ and $\beta$ such that for all $x$ and $y \in \mathbb{R}^{n}$ we have

$$
\alpha\|x-y\|_{\infty} \leq d_{g}(x, y) \leq \beta\|x-y\|_{\infty} ;
$$

thus for every $x \in B_{\infty}$ (1) take the closest point $x_{i}$ (thus $\gamma_{i}$ is the closest point of $\mathbb{Z}^{n} \cdot 0$ from $r x$ ) then there is a constant $C$ (the diameter of the fundamental domain) such that

$$
\left\|x-x_{i}\right\|_{\infty} \leq \frac{1}{\alpha r} d_{g}\left(r x, \gamma_{i}\right) \leq \frac{1}{\alpha r} C
$$

we also get

$$
d_{\rho}\left(x, x_{i}\right) \leq \frac{\beta}{\alpha r} C
$$

thus, for $r$ large enough $\left(x_{1}, \ldots, x_{N}\right)$ is an $\epsilon$-neighbourhood of $\left(B_{\infty}(1)\right.$, $\left.\|\cdot\|_{\infty}\right)$. Furthermore if $\rho$ is large enough it is also an $\epsilon$-neighbourhood of $\left(B_{\rho}(1), d_{\rho}\right)$ and by Corollary 9

$$
\left|\left\|x_{i}-x_{j}\right\|_{\infty}-d_{\rho}\left(x_{i}, x_{j}\right)\right| \leq \epsilon .
$$

Now let us take a continuous function from $B_{\infty}(1)$ to $\mathbb{R}$. Let $z_{1}, \ldots, z_{k}$ and $\zeta_{1}, \ldots, \zeta_{l}$ in the orbit of 0 by the $\mathbb{Z}^{n}$ action such that $\zeta_{j}+D \cap B_{\infty}(\rho) \neq \emptyset$ for $j=1, \ldots l$ and

$$
\bigcup_{i} z_{i}+D_{f} \subset B_{\infty}(\rho) \subset \bigcup_{k} \zeta_{k}+D_{f}
$$

(where we took all $z_{i}$ such that $z_{i}+D_{f} \subset B_{\infty}(\rho)$ ) then we get

$$
\begin{aligned}
\sum_{i} \inf _{\rho x \in z_{i}+D_{f}} f(x) \mu_{\infty}\left(D_{f}\right) & \leq \int_{B_{\infty}(\rho)} f(x / \rho) d \mu_{g}(x) \\
& \leq \sum_{j} \sup _{\rho x \in\left(\zeta_{j}+D_{f}\right) \cap B_{\infty}(\rho)} f(x) \mu_{\infty}\left(D_{f}\right)
\end{aligned}
$$


now dividing by $\rho^{n}$ we find

$$
\begin{aligned}
& \sum_{i} \inf _{x \in \frac{1}{\rho}\left(z_{i}+D_{f}\right)} f(x) \mu_{\infty}\left((1 / \rho) D_{f}\right) \\
& \leq \int_{B_{\infty}(1)} f d \mu_{\rho}(x) \\
& \leq \sum_{j} \sup _{x \in \frac{1}{\rho}\left(\zeta_{j}+D_{f}\right) \cap B_{\infty}(1)} f(x) \mu_{\infty}\left((1 / \rho) D_{f}\right) .
\end{aligned}
$$

The middle term is surrounded by two sums of Riemann, which converges to $\int_{B_{\infty}(1)} f d \mu_{\infty}$, thus it also converges. To conclude, notice that the net of characteristic function $\chi_{B_{\rho}(1)}$ converges simply to $\chi_{B_{\infty}(1)}$ inside of $B_{\infty}(1)$.

2.4. What shall we finally study? As we said we are now going to focus on the spectrum of the balls $B_{g}(\rho)$. As we already mentioned we know that the eigenvalues are converging to zero with a $1 / \rho^{2}$ speed. Hence we want to find a precise equivalent.

For this let introduce $\Delta_{\rho}$ the Laplacian associated to the rescaled metrics $g_{\rho}=1 / \rho^{2}\left(\delta_{\rho}\right)^{*} g$, and for any function $f$ from $B_{g}(\rho)$ to $\mathbb{R}$ lets associate a function $f_{\rho}$ on $B_{\rho}(1)$ by $f_{\rho}(x)=f(\rho \cdot x)$. Then it is an easy computation to see that for any $x \in B_{\rho}(1)$ :

$$
\rho^{2}(\Delta f)(\rho \cdot x)=\left(\Delta_{\rho} f_{\rho}\right)(x)
$$

hence the eigenvalues of $\Delta_{\rho}$ on $B_{\rho}(1)$ are exactly the eigenvalues of $\Delta$ on $B_{g}(\rho)$ multiplied by $\rho^{2}$ and our problems becomes the study of the spectrum of the Laplacian $\Delta_{\rho}$ on $B_{\rho}(1)$. In the light of what precedes we would like to show that there is some operator $\Delta_{\infty}$ acting on $B_{\infty}(1)$ such that, in some sense, the net of Laplacian $\left(\Delta_{\rho}\right)$ converges towards $\Delta_{\infty}$ such that the spectra also converge to the spectrum of $\Delta_{\infty}$. The next section aims at giving a precise meaning to this.

\section{Convergence of spectral nets.}

This section adapts to our purpose some notion of convergences well-known for a fixed Hilbert space.

3.1. Convergence on a net of Hilbert spaces. Let $\left(X_{\alpha}, d_{\alpha}, m_{\alpha}\right)_{\alpha \in \mathcal{A}}$, where $\mathcal{A}$ is a partially ordered set, be a net of compact measured metric spaces converging to $\left(X_{\infty}, d_{\infty}, m_{\infty}\right)$ in the Gromov-Hausdorff measured topology. We will write $L_{\alpha}^{2}=L^{2}\left(X_{\alpha}, m_{\alpha}\right)$ (resp. $\left.L_{\infty}^{2}\left(X_{\infty}, m_{\infty}\right)\right)$ for the square integrable function spaces. Their respective scalar product will be $\langle\cdot, \cdot\rangle_{\alpha}\left(\operatorname{resp} .\langle\cdot, \cdot\rangle_{\infty}\right)$ and $\|\cdot\|_{\alpha}\left(\right.$ resp. $\left.\|\cdot\|_{\infty}\right)$.

Furthermore we suppose that in every $L_{\alpha}^{2}$ the continuous functions form a dense subset. 
Definition 11. We say that a net $\left(u_{\alpha}\right)_{\alpha \in \mathcal{A}}$ of functions $u_{\alpha} \in L_{\alpha}^{2}$ strongly converges to $u \in L_{\infty}^{2}$ if there exists a net $\left(v_{\beta}\right)_{\beta \in \mathcal{B}} \subset C^{0}\left(X_{\infty}\right)$ converging to $u$ in $L_{\infty}^{2}$ such that

$$
\lim _{\beta} \lim \sup \left\|f_{\alpha}^{*} v_{\beta}-u_{\alpha}\right\|_{\alpha}=0
$$

where $\left(f_{\alpha}\right)$ is the net of Hausdorff approximations. We will also talk of strong convergence in $\mathcal{L}^{2}$.

Definition 12. We say that a net $\left(u_{\alpha}\right)_{\alpha \in \mathcal{A}}$ of functions $u_{\alpha} \in L_{\alpha}^{2}$ weakly converges to $u \in L_{\infty}^{2}$ if and only if for every net $\left(v_{\alpha}\right)_{\alpha \in \mathcal{A}}$ strongly converging to $v \in L_{\infty}^{2}$ we have

$$
\lim _{\alpha}\left\langle u_{\alpha}, v_{\alpha}\right\rangle_{\alpha}=\langle u, v\rangle_{\infty}
$$

We will also talk of weak convergence in $\mathcal{L}^{2}$.

The following lemmas justify those two definitions:

Lemma 13. Let $\left(u_{\alpha}\right)_{\alpha \in \mathcal{A}}$ be a net of functions $u_{\alpha} \in L_{\alpha}^{2}$. If $\left(\left\|u_{\alpha}\right\|_{\alpha}\right)$ is uniformly bounded, then there exists a weakly converging subnet.

Proof. Let $\left(\phi_{k}\right)_{k \in \mathbb{N}}$ be a complete orthonormal basis of $L_{\infty}^{2}$. Using the density of continuous functions in $L_{\infty}^{2}$, for each $k$ we can retrieve a net of continuous functions $\left(\varphi_{k, \beta}\right)_{\beta \in \mathcal{B}}$ strongly converging to $\phi_{k}$ in $L_{\infty}^{2}$. Replacing by a subnet of $\mathcal{A}$ and $\mathcal{B}$ if necessarily, we can assume that the following limit exists:

$$
\lim _{\beta} \lim _{\alpha}\left\langle u_{\alpha}, f_{\alpha}^{*} \varphi_{1, \beta}\right\rangle_{\alpha}=a_{1} \in \overline{\mathbb{R}}
$$

and from the uniform bound hypothesis it follows that $a_{1} \in \mathbb{R}$. Repeating this procedure we can assume that for every $k \in \mathbb{N}$ the following limit exists:

$$
\lim _{\beta} \lim _{\alpha}\left\langle u_{\alpha}, f_{\alpha}^{*} \varphi_{k, \beta}\right\rangle_{\alpha}=a_{k} \in \mathbb{R} .
$$

Let us fix an integer $N$. For any $\epsilon>0$ there is a $\beta_{\epsilon} \in \mathcal{B}$ such that

$$
\left|\left\langle\varphi_{k, \beta}, \varphi_{l, \beta}\right\rangle_{\infty}-\delta_{k l}\right|<\epsilon
$$

for any $\beta \geq \beta_{\epsilon}$ and $k, l=1, \ldots, N$. Moreover for any $\beta \geq \beta_{\epsilon}$ there is an $\alpha_{\epsilon, \beta} \in \mathcal{A}$ such that

$$
\left|\left\langle f_{\alpha}^{*} \varphi_{k, \beta}, f_{\alpha}^{*} \varphi_{l, \beta}\right\rangle_{\alpha}-\delta_{k l}\right|<2 \epsilon
$$

for any $\alpha \geq \alpha_{\epsilon, \beta}$ and $k, l=1, \ldots, N$. Let $L_{\alpha, \beta}=\operatorname{Vect}\left\{f_{\alpha}^{*} \varphi_{k, \beta} \mid k=\right.$ $1, \ldots, N\}$ and $P_{\alpha, \beta}: L_{\alpha}^{2} \rightarrow L_{\alpha, \beta}$ be the projection to the linear subspace $L_{\alpha, \beta} \subset L_{\alpha}^{2}$ we have

$$
\left.\left|\sum_{k=1}^{N}\right|\left\langle u_{\alpha}, f_{\alpha}^{*} \varphi_{k, \beta}\right\rangle_{\alpha}\right|^{2}-\left\|P_{\alpha, \beta} u_{\alpha}\right\|_{\alpha}^{2} \mid \leq \theta_{N}(\epsilon)
$$


for every $\alpha \geq \alpha_{\epsilon, \beta}$ and $\beta \geq \beta_{\epsilon}$, where $\theta_{N}$ is a function depending only of $N$ such that $\lim _{\epsilon \rightarrow 0} \theta_{N}(\epsilon)=0$. This implies for every $N$

$$
\begin{aligned}
\sum_{k=1}^{N}\left|a_{k}\right|^{2} & =\lim _{\beta} \lim _{\alpha} \sum_{k=1}^{N}\left|\left\langle u_{\alpha}, f_{\alpha}^{*} \varphi_{k, \beta}\right\rangle_{\alpha}\right|^{2}=\lim _{\beta} \lim _{\alpha}\left\|P_{\alpha, \beta} u_{\alpha}\right\|_{\alpha}^{2} \\
& \leq \limsup _{\alpha}\left\|u_{\alpha}\right\|_{\alpha}^{2}<\infty
\end{aligned}
$$

thus

$$
u=\sum_{k=1}^{N} a_{k} \phi_{k} \in L_{\infty}^{2} .
$$

We shall prove that some subnet of $\left(u_{\alpha}\right)_{\alpha}$ weakly converges to $u$. Take any $v \in L_{\infty}^{2}$ and set $b_{k}=\left\langle v, \phi_{k}\right\rangle_{\infty}$. By the properties of the strong convergence it is enough to show (3) for a well chosen net. Let $v_{\beta}^{N}=\sum_{k=1}^{N} b_{k} \varphi_{k, \beta}$. By construction $v_{\beta}^{N} \in \mathcal{C}^{0}$ and $\lim _{N \rightarrow \infty} \lim _{\beta} v_{\beta}^{N}=v$ strongly. We have

$$
\lim _{\beta} \lim _{\alpha}\left\langle u_{\alpha}, f_{\alpha}^{*} v_{\beta}^{N}\right\rangle_{\alpha}=\lim _{\beta} \lim _{\alpha} \sum_{k=1}^{N} b_{k}\left\langle u_{\alpha}, f_{\alpha}^{*} \varphi_{k, \beta}\right\rangle_{\alpha}=\sum_{k=1}^{N} a_{k} b_{k}
$$

which tends to $\langle u, v\rangle_{\infty}$ as $N \rightarrow \infty$. Thus, there exists a net of integers $\left(N_{\beta}\right)_{\beta}$ tending to $+\infty$ such that $v_{\beta}^{N_{\beta}}$ strongly converges to $v$ and

$$
\lim _{\beta} \lim _{\alpha}\left\langle u_{\alpha}, f_{\alpha}^{*} v_{\beta}^{N_{\beta}}\right\rangle_{\alpha}=\langle u, v\rangle_{\infty}
$$

Lemma 14. Let $\left(u_{\alpha}\right)_{\alpha \in \mathcal{A}}$ be a weakly converging net to $u \in L_{\infty}^{2}$. Then

$$
\sup _{\alpha}\left\|u_{\alpha}\right\|_{\alpha}<\infty \quad \text { and } \quad\|u\|_{\infty} \leq \liminf _{\alpha}\left\|u_{\alpha}\right\|_{\alpha} \text {. }
$$

Furthermore, the net strongly converges if and only if

$$
\|u\|_{\infty}=\lim _{\alpha}\left\|u_{\alpha}\right\|_{\alpha} .
$$

Proof. Let suppose that the net $\left(u_{\alpha}\right)$ is weakly converging and $\sup _{\alpha}\left\|u_{\alpha}\right\|_{\alpha}=$ $+\infty$. We can extract a sequence such that $\left\|u_{\alpha_{k}}\right\|_{\alpha_{k}}>k$. Setting

$$
v_{k}=\frac{1}{k} \frac{u_{\alpha_{k}}}{\left\|u_{\alpha_{k}}\right\|_{\alpha_{k}}}
$$

one has $\left\|v_{k}\right\|_{\alpha_{k}}=1 / k \rightarrow 0$ thus $v_{k}$ strongly converges to 0 , which implies

$$
\left\langle u_{\alpha_{k}}, v_{k}\right\rangle_{\alpha_{k}} \rightarrow\langle u, 0\rangle_{\infty}=0
$$

but we also have

$$
\left\langle u_{\alpha_{k}}, v_{k}\right\rangle_{\alpha_{k}}=\frac{1}{k}\left\|u_{\alpha_{k}}\right\|_{\alpha_{k}} \geq 1
$$

this is a contradiction and thus we obtain $\sup _{\alpha}\left\|u_{\alpha}\right\|_{\alpha}<\infty$. 
Let $\left(w_{\alpha}\right)_{\alpha}$ be a strongly converging net to $u$, then

$$
\begin{aligned}
0 & \leq \liminf _{\alpha}\left\|u_{\alpha}-w_{\alpha}\right\|_{\alpha}^{2} \\
& =\liminf _{\alpha}\left(\left\|u_{\alpha}\right\|_{\alpha}^{2}+\left\|w_{\alpha}\right\|_{\alpha}^{2}-2\left\langle u_{\alpha}, w_{\alpha}\right\rangle_{\alpha}\right) \\
& =\liminf _{\alpha}\left\|u_{\alpha}\right\|_{\alpha}^{2}-\|u\|_{\infty}^{2} .
\end{aligned}
$$

The final claim comes from the properties of the strong convergence and the following equality:

$$
\left\|u_{\alpha}-w_{\alpha}\right\|_{\alpha}^{2}=\left\|u_{\alpha}\right\|_{\alpha}^{2}+\left\|w_{\alpha}\right\|_{\alpha}^{2}-2\left\langle u_{\alpha}, w_{\alpha}\right\rangle_{\alpha} .
$$

3.2. Convergence of bounded operators. Let $\mathcal{L}\left(L_{\alpha}^{2}\right)$ bet the set of linear bounded operators acting on $L_{\alpha}^{2}$ and $\|\cdot\|_{\mathcal{L}_{\alpha}}$ their norm (for $\alpha \in \mathcal{A} \cup \infty$ ). Let $B_{\infty} \in \mathcal{L}\left(L_{\infty}^{2}\right)$ and $B_{\alpha} \in \mathcal{L}\left(L_{\alpha}^{2}\right)$ for every $\alpha \in \mathcal{A}$.

Theorem and Definition 15. Let $u, v \in L_{\infty}^{2}$ and $\left(u_{\alpha}\right)_{\alpha \in \mathcal{A}},\left(v_{\alpha}\right)_{\alpha \in \mathcal{A}}$ two nets such that $u_{\alpha}, v_{\alpha} \in L_{\alpha}^{2}$. We say that the net of operators $\left(B_{\alpha}\right)_{\alpha \in \mathcal{A}}$ strongly (resp. weakly, compactly) converges to $B$ if $B_{\alpha} u_{\alpha} \rightarrow B u$ strongly (resp. weakly, strongly) for every net $\left(u_{\alpha}\right)$ strongly (resp. weakly, weakly) converging to $u \Longleftrightarrow$

$$
\lim _{\alpha}\left\langle B_{\alpha} u_{\alpha}, v_{\alpha}\right\rangle_{\alpha}=\langle B u, v\rangle_{\infty}
$$

for every $\left(u_{\alpha}\right),\left(v_{\alpha}\right), u$ and $v$ such that $u_{\alpha} \rightarrow u$ strongly (resp. weakly, weakly) and $v_{\alpha} \rightarrow v$ weakly (resp. strongly, weakly).

Proof. The equivalence comes from the definition of the weak convergence and the fact that a net $\left(u_{\alpha}\right)$ strongly converges to $u$ if and only if $\left\langle u_{\alpha}, v_{\alpha}\right\rangle_{\alpha} \rightarrow$ $\langle u, v\rangle_{\infty}$ for every net $\left(v_{\alpha}\right)_{\alpha}$ weakly converging to $v \in L_{\infty}^{2}$. The "if" part is straightforward, for the "only if" we see that for every net $\left(v_{\alpha}\right)$ strongly converging to $v$ we have $\left\langle u_{\alpha}, v_{\alpha}\right\rangle_{\alpha} \rightarrow\langle u, v\rangle_{\infty}$, which implies the weak convergence of the net $\left(u_{\alpha}\right)$. Using now the hypothesis we get the convergence of the net $\left\|u_{\alpha}\right\|_{\alpha}$ and thus the strong convergence of $\left(u_{\alpha}\right)$ by Lemma 14 .

Proposition 16. Let $\left(B_{\alpha}\right)$ be a strongly converging net to $B$ then

$$
\liminf _{\alpha}\left\|B_{\alpha}\right\|_{\mathcal{L}_{\alpha}} \geq\|B\|_{\mathcal{L}_{\infty}}
$$

and if the convergence is compact then it is an equality and $B$ is a compact operator as is its adjoint $B^{*}$.

Proof. Let $\epsilon>0$, there is $u \in \mathcal{L}_{\infty}^{2}$ such that $\|u\|_{\infty}=1$ and $\|B u\|_{\infty}>$ $\|B\|_{\mathcal{L}_{\infty}}-\epsilon$. Take $\left(u_{\alpha}\right)_{\alpha}$ a net converging strongly to $u$. Then $\left\|u_{\alpha}\right\|_{\alpha} \rightarrow 1$, furthermore the strong convergence of $\left(B_{\alpha}\right)$ implies that $\left\|B_{\alpha} u_{\alpha}\right\|_{\alpha} \rightarrow\|B u\|_{\infty}$ thus

$$
\liminf _{\alpha}\left\|B_{\alpha}\right\|_{\mathcal{L}_{\alpha}} \geq \liminf _{\alpha} \frac{\left\|B_{\alpha} u_{\alpha}\right\|_{\alpha}}{\left\|u_{\alpha}\right\|_{\alpha}}=\|B u\|_{\infty}>\|B\|_{\mathcal{L}_{\infty}}-\epsilon
$$


Suppose now that the convergence is compact. Take a net $\left(u_{\alpha}\right)$ such that $\left\|u_{\alpha}\right\|_{\alpha}=1$ and

$$
\lim _{\alpha}\left|\left\|B_{\alpha}\right\|_{\mathcal{L}_{\alpha}}-\left\|B_{\alpha} u_{\alpha}\right\|_{\alpha}\right|=0
$$

Extracting a subnet if necessary we can suppose that the net $\left(u_{\alpha}\right)$ weakly converges to $u$. By Lemma 14 we have $\|u\|_{\infty} \leq 1$, furthermore the compact convergence implies the strong convergence of $B_{\alpha} u_{\alpha}$ to $B u$ thus

$$
\|B\|_{\mathcal{L}_{\infty}} \geq \frac{\|B u\|_{\infty}}{\|u\|_{\infty}} \geq\|B u\|_{\infty}=\lim _{\alpha}\left\|B_{\alpha} u_{\alpha}\right\|_{\alpha}=\lim _{\alpha}\left\|B_{\alpha}\right\|_{\mathcal{L}_{\alpha}} .
$$

Now let us prove that in the latest case, $B$ is compact. Let $\left(v_{\beta}\right)_{\beta \in \mathcal{B}}$ a net weakly converging to $v$ in $\mathcal{L}_{\infty}^{2}$ then

$$
\left\langle u, B v_{\beta}\right\rangle_{\infty}=\left\langle B^{*} u, v_{\beta}\right\rangle_{\infty} \rightarrow\left\langle B^{*} u, v\right\rangle_{\infty}=\langle u, B v\rangle_{\infty}
$$

thus $B v_{\beta}$ weakly converges to $B v$. For every $\beta$ let $\left(u_{\alpha, \beta}\right)$ be a strongly converging net such that $\lim _{\alpha} u_{\alpha, \beta}=v_{\beta}$. For every $\beta$ the compact convergence of $\left(B_{\alpha}\right)$ implies the strong convergence of $B_{\alpha} u_{\alpha, \beta}$ to $B v_{\beta}$. Now let us take a net of positive numbers such that $\lim _{\beta} \epsilon(\beta)=0$, then there is $\alpha(\beta)$ such that for every $\alpha \geq \alpha(\beta)$ we have

$$
\left|\left\|B_{\alpha} u_{\alpha, \beta}\right\|_{\alpha}-\left\|B v_{\beta}\right\|_{\infty}\right| \leq \epsilon(\beta) .
$$

Set $w_{\beta}=u_{\alpha(\beta), \beta}$ then $\lim _{\beta} w_{\beta}=v$ weakly and by the compact convergence we obtain the strong convergence of $\left(B_{\alpha(\beta)} w_{\beta}\right)_{\beta}$ to $B v$ but

$$
\lim _{\beta}\left|\left\|B_{\alpha(\beta)} w_{\beta}\right\|_{\alpha(\beta)}-\left\|B v_{\beta}\right\|_{\infty}\right|=0
$$

which implies $\left\|B v_{\beta}\right\|_{\infty} \rightarrow\|B v\|_{\infty}$. We can conclude using Lemma 14 .

3.3. Convergence of spectral structures. Here we see $L_{\alpha}^{2}$ as a Hilbert space. Then $A_{\alpha}$ and $A$ will be self-adjoint operators, $E_{\alpha}$ and $E$ their respective spectral measure and $R_{\mu}, R$ their resolvents for $\mu$ in the resolvent space. We want to study the links between the convergence of $\left(A_{\alpha}\right),\left(E_{\alpha}\right)$ and $\left(R_{\mu}^{\alpha}\right)$. The following theorem says that it is the same:

Theorem 17. Let $\left(A_{\alpha}\right)$ and $A$ be self-adjoint operators $E_{\alpha}, E$ their spectral measures and $R_{\mu}^{\alpha}, R_{\mu}$ their resolvents for $\mu$ in the resolvent space, then the following assertions are equivalent:

(1) $R_{\mu}^{\alpha} \rightarrow R_{\mu}$ strongly (resp. compactly) for $\mu$ outside the union of the spectra of $A_{\alpha}$ and $A$.

(2) $\varphi\left(A_{\alpha}\right) \rightarrow \varphi(A)$ strongly (resp. compactly) for every continuous function, with compact support $\varphi: \mathbb{R} \rightarrow \mathbb{C}$.

(3) $\varphi_{\alpha}\left(A_{\alpha}\right) \rightarrow \varphi(A)$ strongly (resp. compactly) for every net $\left\{\varphi_{\alpha}: \mathbb{R} \rightarrow \mathbb{C}\right\}$ of continuous functions vanishing at infinity and uniformly converging to $\varphi$ a continuous function vanishing at infinity.

(4) $\left.\left.\left.\left.E_{\alpha}(] \lambda, \mu\right]\right) \rightarrow E(] \lambda, \mu\right]\right)$ strongly (resp. compactly) for every pair of real numbers outside the spectrum of $A$. 
(5) $\left\langle E_{\alpha} u_{\alpha}, v_{\alpha}\right\rangle_{\alpha} \rightarrow\langle E u, v\rangle_{\infty}$ vaguely for every net of vectors $\left(u_{\alpha}\right)_{\alpha \in \mathcal{A}}$ and $\left(v_{\alpha}\right)_{\alpha \in \mathcal{A}}$ such that $u_{\alpha} \rightarrow u$ strongly (resp. weakly) and $v_{\alpha} \rightarrow v$ weakly.

Let us recall that a quadratic form $\mathcal{Q}$ on a complex (resp. real) Hilbert space $\mathcal{H}$ comes from a sesquilinear (resp. bilinear) form, positive and symmetric $\mathcal{E}: D(\mathcal{E}) \times D(\mathcal{E}) \rightarrow \mathbb{C}($ resp. $\mathbb{R})$ where $D(E) \in \mathcal{H}$ is a linear subspace and $\mathcal{Q}(u)=\mathcal{E}(u, u)$. Notice that $\mathcal{E}_{1}(u, v)=\langle u, v\rangle_{\mathcal{H}}+\mathcal{E}(u, v)$ for every $u$ and $v \in D(\mathcal{E})$ is also a sesquilinear (resp. bilinear), symmetric and positive form. Thus $\left(D(\mathcal{E}), \mathcal{E}_{1}\right)$ is a pre-Hilbert space. We say that $\mathcal{Q}$ is closed if and only if $\left(D(\mathcal{E}), \mathcal{E}_{1}\right)$ is a Hilbert space. In what follows, we will not distinguish $\mathcal{Q}$ and the functional $\mathcal{E}$ defined by $\mathcal{E}(u)=\mathcal{Q}(u)$ on $D(\mathcal{E})$ and $\mathcal{E}(u)=\infty$ on $\mathcal{H} \backslash D(\mathcal{E})$. In this context, $\mathcal{Q}$ is closed if and only if $\mathcal{E}$ is lower semi-continuous as a function $\mathcal{E}: \mathcal{H} \rightarrow \overline{\mathbb{R}}$.

Definition 18. Let $\left(\mathcal{E}_{\alpha}\right)$ be a net of closed quadratic forms, where $\mathcal{E}_{\alpha}$ is a closed quadratic form on $L_{\alpha}^{2}$ for every $\alpha \in \mathcal{A}$. We will say that this net is asymptotically compact if and only if for every net $\left(v_{\alpha}\right)_{\alpha \in \mathcal{A}}$ such that

$$
\limsup _{\alpha} \mathcal{E}_{\alpha}\left(v_{\alpha}\right)+\left\|v_{\alpha}\right\|_{\alpha}^{2}<\infty
$$

there is a strongly converging subnet.

Now a spectral structure on a Hilbert space $\mathcal{H}$ over $\mathbb{C}($ resp. $\mathbb{R})$ is a family

$$
\Sigma=\left\{A, \mathcal{E}, E,\left(T_{t}\right),\left(R_{\zeta}\right)\right\}
$$

where $A$ is a self-adjoint operator seen as the infinitesimal generator of the densely defined quadratic form $\mathcal{E}$ (such that $D(\mathcal{E})=D(\sqrt{A})$ and $\mathcal{E}(u, v)=$ $\langle\sqrt{A} u, \sqrt{A} v\rangle_{\mathcal{H}}$ for every $u$ and $v$ in $\left.D(\mathcal{E})\right), E$ is its spectral measure, $\left(T_{t}\right)_{t \geq 0}$ is a one parameter semi-group of strongly continuous contractions $\left(T_{t}=e^{-t A}\right.$, $t \geq 0)$ and $R_{\zeta}$ is a strongly continuous resolvent $\left(R_{\zeta}=(\zeta-A)^{-1}\right.$ for $\zeta \in \rho(A)$, where $\rho(A)$ is the resolvent set of $A$ ). In what follows we will study a family of spectral structures $\Sigma_{\alpha}$ on $L_{\alpha}^{2}$, thus we will have

$$
\Sigma_{\alpha}=\left\{A_{\alpha}, \mathcal{E}_{\alpha}, E_{\alpha},\left(T_{t}^{\alpha}\right),\left(R_{\zeta}^{\alpha}\right)\right\}
$$

Definition 19. Let $\left(\Sigma_{\alpha}\right)_{\alpha \in \mathcal{A}}$ be a net with $\Sigma_{\alpha}$ a spectral structure on $L_{\alpha}^{2}$ and $\Sigma$ a spectral structure on $L_{\infty}^{2}$, we will say that the net $\left(\Sigma_{\alpha}\right)_{\alpha}$ strongly (resp. compactly) converges to $\Sigma$ if and only if one of the conditions of Theorem 17 is satisfied.

Proposition 20. Let $\left(\Sigma_{\alpha}\right)_{\alpha \in \mathcal{A}}$ of spectral structures strongly converging to $\Sigma$ then for any net $\left(v_{\alpha}\right)_{\alpha}$ weakly converging to $v$ we have

$$
\mathcal{E}(v) \leq \liminf _{\alpha} \mathcal{E}_{\alpha}\left(v_{\alpha}\right)
$$

Furthermore, if the net $\left(\Sigma_{\alpha}\right)_{\alpha \in \mathcal{A}}$ converges compactly, then the net of quadratic forms $\left(\mathcal{E}_{\alpha}\right)_{\alpha}$ is asymptotically compact. 
Proof. Assume that the net of resolvents $\left(R_{\lambda}^{\alpha}\right)$ is strongly convergent and write

$$
a_{\alpha}^{\lambda}(u, v)=-\lambda\left\langle u-\lambda R_{\lambda}^{\alpha} u, v\right\rangle_{\alpha}
$$

(the Deny-Yosida approximation of bilinear form associated to $\mathcal{E}_{\alpha}$ ), then the net $\left(a_{\alpha}^{\lambda}(u, u)\right)$ converges to $\mathcal{E}_{\alpha}(u)$ increasing when $\lambda \rightarrow-\infty$ (see Mosco [Mos94] 1.(i)). From the assumption it easy to see that for $\left(u_{\alpha}\right)$ and $\left(v_{\alpha}\right)$ converging strongly to $u$ and weakly to $v$ respectively

$$
\lim _{\alpha} a_{\alpha}^{\lambda}\left(u_{\alpha}, v_{\alpha}\right)=-\lambda\left\langle u-\lambda R_{\lambda} u, v\right\rangle_{\infty}=a^{\lambda}(u, v)
$$

we recall that (see Dal Maso [Mas93] Proposition 12.12)

$$
a^{\lambda}(u, u) \geq a^{\lambda}(v, v)+2 \lambda\left\langle v-\lambda R_{\lambda} v, u-v\right\rangle_{\infty}
$$

hence for any net $v_{\alpha}$ weakly converging to $u$ and $w_{\alpha}$ a strongly converging net to $u$ we have

$$
\mathcal{E}_{\alpha}\left(v_{\alpha}\right) \geq a_{\alpha}^{\lambda}\left(v_{\alpha}, v_{\alpha}\right) \geq a_{\alpha}^{\lambda}\left(w_{\alpha}, w_{\alpha}\right)+2 \lambda\left\langle w_{\alpha}-\lambda R_{\lambda}^{\alpha} w_{\alpha}, v_{\alpha}-w_{\alpha}\right\rangle
$$

thus $\liminf \operatorname{in}_{\alpha} \mathcal{E}_{\alpha}\left(v_{\alpha}\right) \geq a^{\lambda}(u, u)$ for any $\lambda<0$, now taking $\lambda \rightarrow-\infty$ we can conclude that $\liminf _{\alpha} \mathcal{E}_{\alpha}\left(v_{\alpha}\right) \geq \mathcal{E}(u)$.

Now assume that $\left(\Sigma_{\alpha}\right)$ compactly converges and let $\left(u_{\alpha}\right)_{\alpha \in \mathcal{A}}$ be a net such that

$$
\sup _{\alpha}\left(\mathcal{E}_{\alpha}\left(u_{\alpha}\right)+\left\|u_{\alpha}\right\|_{\alpha}^{2}\right) \leq M<\infty .
$$

Taking a subnet if necessary we can suppose that $\left(u_{\alpha}\right)_{\alpha}$ weakly converges to $u$. Let $\rho>0$ be out of $A_{\infty}$ 's spectrum. As

$$
\int_{] \rho, \infty[} d\left\langle E_{\alpha} u_{\alpha}, u_{\alpha}\right\rangle_{\alpha} \leq \frac{1}{\rho} \int_{] \rho, \infty[} \lambda d\left\langle E_{\alpha}(\lambda) u_{\alpha}, u_{\alpha}\right\rangle_{\alpha} \leq \frac{\mathcal{E}_{\alpha}\left(u_{\alpha}\right)}{\rho} \leq \frac{M}{\rho}
$$

we have

$$
\left\|u_{\alpha}\right\|_{\alpha}^{2} \leq \int_{[0, \rho]} d\left\langle E_{\alpha} u_{\alpha}, u_{\alpha}\right\rangle_{\alpha}+\frac{M}{\rho}
$$

and the compact convergence implies

$$
\lim _{\alpha} \int_{[0, \rho]} d\left\langle E_{\alpha} u_{\alpha}, u_{\alpha}\right\rangle_{\alpha}=\int_{[0, \rho]} d\langle E u, u\rangle_{\infty}
$$

hence

$$
\limsup _{\alpha}\left\|u_{\alpha}\right\|_{\alpha}^{2} \leq \int_{[0, \rho]} d\langle E u, u\rangle_{\infty}+\frac{M}{\rho} \leq\|u\|_{\infty}^{2}+\frac{M}{\rho}
$$

and taking $\rho \rightarrow \infty$ we get

$$
\limsup \left\|u_{\alpha}\right\|_{\alpha}^{2} \leq\|u\|_{\infty}^{2}
$$

finally we deduce the strongly convergence of the net $\left(u_{\alpha}\right)$ using Lemma 14 . 
The main reason we introduced all these convergences is the following theorem, the proof of which we postpone to avoid drowning the reader in too many technical details.

Theorem 21. Let $\Sigma_{\alpha} \rightarrow \Sigma$ compactly and suppose that all resolvents $R_{\zeta}^{\alpha}$ are compact. Let $\lambda_{k}\left(\right.$ resp. $\left.\lambda_{k}^{\alpha}\right)$ be the $k^{\text {th }}$ eigenvalue of $A$ (resp. $\left.A_{\alpha}\right)$ with multiplicity. We take $\lambda_{k}=+\infty$ if $k>\operatorname{dim} L_{\infty}^{2}+1$ when $\operatorname{dim} L_{\infty}^{2}<\infty$ and $\lambda_{k}^{\alpha}=+\infty$ if $k>\operatorname{dim} L_{\alpha}^{2}+1$ when $\operatorname{dim} L_{\alpha}^{2}<\infty$. Then for every $k$

$$
\lim _{\alpha} \lambda_{k}^{\alpha}=\lambda_{k} \text {. }
$$

Furthermore let $\left\{\varphi_{k}^{\alpha} \mid k=1, \ldots, \operatorname{dim} L_{\alpha}^{2}\right\}$ be an orthonormal bases of $L_{\alpha}^{2}$ such that $\varphi_{k}^{\alpha}$ is an eigenfunction of $A_{\alpha}$ for $\lambda_{k}^{\alpha}$. Then there is a subnet such that for all $k \leq \operatorname{dim} L_{\infty}^{2}$ the net $\left(\varphi_{k}^{\alpha}\right)_{\alpha}$ strongly converges to the eigenfunction $\varphi_{k}$ of $A$ for the eigenvalue $\lambda_{k}$, and such that the family $\left\{\varphi_{k} \mid k=\right.$ $\left.1, \ldots, \operatorname{dim} L_{\alpha}^{2}\right\}$ is an orthonormal basis of $L_{\infty}^{2}$.

\section{Proof of Theorem 1.}

4.1. Homogenisation of the Laplacian. In this section we are going to built the operator $\Delta_{\infty}$ of Theorem 1 . We remind the reader that $D_{f}$ is a fundamental domain, we then begin by taking $\chi^{i}$ as the unique periodic solution (up to an additive constant) of

$$
\Delta \chi^{i}=\Delta x_{i} \text { on } D_{f} \text {. }
$$

The operator $\Delta_{\infty}$ is then defined by

$$
\Delta_{\infty} f=-\frac{1}{\operatorname{Vol}(g)}\left(\int_{D_{f}} g^{i j}-g^{i k} \frac{\partial \chi^{j}}{\partial y_{k}} d \mu_{g}\right) \frac{\partial^{2} f}{\partial x_{i} \partial x_{j}} .
$$

Now let us write $\eta_{j}(x)=\chi^{j}(x)-x_{j}$ the induced harmonic function and

$$
q^{i j}=d \frac{1}{\operatorname{Vol}(g)}\left(\int_{D_{f}} g^{i j}-g^{i k} \frac{\partial \chi^{j}}{\partial y_{k}} d \mu_{g}\right)
$$

we can notice that the $d \eta_{i}$ are harmonic 1-forms on the torus. It is not difficult now to show that:

Proposition 22. Let $\langle\cdot, \cdot\rangle_{2}$ be the scalar product induced on 1-forms by the Riemannian metric $g$. Then

$$
q^{i j}=\frac{1}{\operatorname{Vol}(g)}\left\langle d \eta_{i}, d \eta_{j}\right\rangle_{2}=q^{j i}
$$

thus $\Delta_{\infty}$ is an elliptic operator.

In fact we can say more, $\left(q^{i j}\right)$ induces a scalar product on harmonic 1forms (whose norm will be written $\|\cdot\|_{2}$ ) and then to $H^{1}(\mathbb{T}, \mathbb{R})$. Indeed, as mentioned earlier, we can see the $\left(d \eta_{i}\right)$ as 1 -forms over the torus. Being a free 
family they can be seen as a basis of $H^{1}(\mathbb{T}, \mathbb{R})$ (Hodge's theorem). Thus by duality this yields also a scalar product $\left(q_{i j}\right)$ over $H_{1}(\mathbb{T}, \mathbb{R})$ (whose induced norm will be written $\left.\|\cdot\|_{2}^{*}\right)$. The question naturally arising is to know the link between this norm and the stable norm. To see this we have to go back on $H^{1}(\mathbb{T}, \mathbb{R})$. Indeed the stable norm is the dual of the norm obtained by quotient of the sup norm on 1-forms (see Pansu [Pan99] Lemma 17), which we write $\|\cdot\|_{\infty}^{*}$, and the norm $\|\cdot\|_{2}$ comes from the normalised $L^{2}$ norm. Thus mixing the Hölder inequality and the Hodge-de Rham theorem we get:

Proposition 23. For every 1-form $\alpha$ we have

$$
\|\alpha\|_{2} \leq\|\alpha\|_{\infty}^{*}
$$

thus by duality, for every $\gamma \in H_{1}(\mathbb{T}, \mathbb{R})$ we have

$$
\|\gamma\|_{\infty} \leq\|\gamma\|_{2}^{*}
$$

in other words the unit ball of $\|\cdot\|_{2}^{*}$ is included in $B_{\infty}(1)$.

To finish this section, let us remark that the manifold $H_{1}(\mathbb{T}, \mathbb{R}) / H_{1}(\mathbb{T}, \mathbb{Z})$ with the flat metric induced by $\|\cdot\|_{2}^{*}$ is usually called the Jacobi manifold or the Albanese torus of $(\mathbb{T}, g)$.

4.2. Asymptotic compactness. Let us now define the various functional spaces involved. For $\rho \in \overline{\mathbb{R}}, L^{2}\left(B_{\rho}(1), d \mu_{\rho}\right)$ will be the space of square integrable functions over the ball $B_{\rho}(1)$, which is a Hilbert space with the scalar product

$$
(u, v)_{\rho}=\int_{B_{\rho}(1)} u v d \mu_{\rho}
$$

whose norm will be $|\cdot|_{\rho}$. $H_{\rho, 0}^{1}\left(B_{\rho}(1)\right)$ will be the closure of $C^{\infty}\left(B_{\rho}(1)\right)$ functions with compact support, in $H_{\rho}^{1}\left(B_{\rho}(1)\right)$ for the norm $\|\cdot\|_{\rho}$ defined by

$$
\|v\|_{\rho}^{2}=|v|_{\rho}^{2}+\sum_{i=1}^{n}\left|\frac{\partial v}{\partial x_{i}}\right|_{\rho}^{2}
$$

and with

$$
H_{\rho}^{1}\left(B_{\rho}(1)\right)=\left\{v \mid v, \frac{\partial v}{\partial x_{1}}, \ldots, \frac{\partial v}{\partial x_{n}} \in L^{2}\left(B_{\rho}(1), d \mu_{\rho}\right)\right\} .
$$

For all that follows, $V_{\rho}$ will be a closed sub-space such that

$$
H_{\rho, 0}^{1}\left(B_{\rho}(1)\right) \subset V_{\rho} \subset H_{\rho}^{1}\left(B_{\rho}(1)\right) .
$$

Thus we can define a spectral structure on $L_{\rho}^{2}$ by expanding the Laplacian defined on $V_{\rho}$ on $L_{\rho}^{2}$. If $V_{\rho}=H_{\rho, 0}^{1}\left(B_{\rho}(1)\right)$ we deal with the Dirichlet problem, and if $V_{\rho}=H_{\rho}^{1}\left(B_{\rho}(1)\right)$ we then deal with the Neumann problem. We then put the following norm on $V_{\rho}$ :

$$
\|v\|_{\rho, 0}^{2}=|v|_{\rho}^{2}+\left(v, \Delta_{\rho} v\right)_{\rho}
$$


we then have:

Lemma 24. Let $\left(u_{\rho}\right)_{\rho}$ be a net with $u_{\rho} \in V_{\rho}$ for every $\rho$, if there is a constant $C$ such that for all $\rho>0$ we have

$$
\left\|u_{\rho}\right\|_{\rho, 0} \leq C
$$

then there is a strongly converging subnet in $\mathcal{L}^{2}$.

Proof. Let $B=\cup_{\rho} B_{\rho}(1)$ we are going to show that the strong convergence in $L^{2}\left(B, \mu_{\infty}\right)$ implies the strong convergence in $\mathcal{L}^{2}$. Then the compact embedding of $H_{\infty}^{1}(B)$ in $L^{2}\left(B, \mu_{\infty}\right)$ will conclude the proof.

Let us first notice that the periodicity gives the existence of two constant $\alpha$ and $\beta$ such that

$$
\alpha|v|_{\infty} \leq|v|_{\rho} \leq \beta|v|_{\infty} .
$$

Let us start by taking a net $\left(u_{\rho}\right)$ strongly converging in $L^{2}\left(B, \mu_{\infty}\right)$ to $u_{\infty}$ we also assume $u_{\rho} \in V_{\rho}$ for every $\rho$, because it is all we need. Now let $c_{p} \in C_{0}^{\infty}\left(B_{\infty}(1)\right)$ be a sequence of functions strongly converging to $u_{\infty}$ and take $p$ large enough for the support of $u_{p}$ to be in $B_{\rho}(1)$. We have

$$
\left|c_{p}-u_{\rho}\right|_{\rho} \leq \beta\left|c_{p}-u_{\infty}\right|_{\infty}+\beta\left|u_{\infty}-u_{\rho}\right|_{\infty}
$$

now let $\varepsilon>0$ then for $p$ large enough $\beta\left|c_{p}-u_{\infty}\right|_{\infty} \leq \varepsilon$. We fix $p$ large enough and take $\rho$ large enough for the second term to converge to 0 .

In order to conclude observe that from the assumptions the net $\left(u_{\rho}\right)$ is bounded in $H_{\infty}^{1}(B)$, hence using the compact embedding of $H_{\infty}^{1}(B)$ in $L^{2}\left(B, \mu_{\infty}\right)$ we can extract a strongly converging net in $L^{2}\left(B, \mu_{\infty}\right)$ and by what we just did in $\mathcal{L}^{2}$.

4.3. Compact convergence of the resolvents. Let $\lambda>0$ and $G_{\lambda}^{\rho}$ be the operator from $L_{\rho}^{2}$ to $V_{\rho} \subset L_{\rho}^{2}$ such that

$$
a_{\lambda}^{\rho}\left(G_{\lambda}^{\rho} f, \phi\right)=(f, \phi)_{\rho} \quad \forall \phi \in V_{\rho}
$$

where

$$
a_{\lambda}^{\rho}(u, v)=\int_{B_{\rho}(1)} g_{\rho}^{i j} \partial_{i} u \cdot \partial_{j} v d \mu_{\rho}+\lambda(u, v)_{\rho} .
$$

We want to show that the net of operators $\left(G_{\lambda}^{\rho}\right)$ converges compactly to $G_{\lambda}$ the operator corresponding to the homogenised problem:

$$
a_{\lambda}^{\infty}\left(G_{\lambda} f, \phi\right)=(f, \phi)_{\infty} \quad \forall \phi \in V_{\infty}
$$

with $(f, \phi)_{\infty}=\int_{B_{\infty}(1)} f \phi d \mu_{\infty}$ and

$$
a_{\lambda}^{\infty}(u, v)=\int_{B_{\infty}(1)} q^{i j} \partial_{i} u \partial_{j} v d \mu_{\infty}+\lambda(u, v)_{\infty}
$$

in other words we want to show the following theorem: 
Theorem 25. For every $\lambda<0$, the net of resolvents $\left(R_{\lambda}^{\rho}\right)_{\rho}$ of the Laplacian $\left(\Delta_{\rho}\right)$ converges compactly to $R_{\lambda}^{\infty}$, the resolvent of $\Delta_{\infty}$ from the homogenised problem. Thus the net $\left(\Sigma_{\rho}\right)$ compactly converges to $\Sigma_{\infty}$.

Proof. This comes from the fact that $R_{\lambda}^{\rho}=-G_{-\lambda}^{\rho}$ and $R_{\lambda}^{\infty}=-G_{-\lambda}$.

First step:

Let $f_{\rho}$ be a weakly convergent net to $f$ in $\mathcal{L}^{2}$, thus from Lemma 14 this net is uniformly bounded in $\mathcal{L}^{2}$ and in $V_{\rho}^{\prime}$, the dual space of $V_{\rho}$.

Let $f_{\rho} \in V_{\rho}$ then by (6) we have:

$$
\alpha\left\|G_{\lambda}^{\rho} f_{\rho}\right\|_{\rho, 0}^{2} \leq\left(f_{\rho}, G_{\lambda}^{\rho} f_{\rho}\right)_{\rho} \leq K\left\|f_{\rho}\right\|_{V_{\rho}^{\prime}}\left\|G_{\lambda}^{\rho} f_{\rho}\right\|_{\rho, 0}
$$

thus

$$
\left\|G_{\lambda}^{\rho} f_{\rho}\right\|_{\rho, 0} \leq C\left\|f_{\rho}\right\|_{V_{\rho}^{\prime}}
$$

the net $\left(G_{\lambda}^{\rho} f_{\rho}\right)$ being uniformly bounded for the norms $\|\cdot\|_{\rho, 0}$, using Lemma 13 there is a subnet strongly converging in $\mathcal{L}^{2}$. i.e.,

$$
u_{\rho}=G_{\lambda}^{\rho} f_{\rho} \rightarrow u_{\lambda}^{*} \text { strongly in } \mathcal{L}^{2} .
$$

Furthermore $P_{\rho}=\left(g_{\rho}^{i j}\right) \nabla G_{\lambda}^{\rho} f_{\rho}$ is also bounded in $\mathcal{L}^{2}$ thus there is a subnet of the net $P_{\rho}$ weakly converging in $\mathcal{L}^{2}$ to $P_{\lambda}^{*} \in L_{\infty}^{2}$. For any $\phi_{\infty} \in L_{\infty}^{2}$ let $\phi_{\rho}$ be a strongly converging net to $\phi_{\infty}$ in $\mathcal{L}^{2}$ then

$$
\begin{aligned}
& \int_{B_{\rho}(1)} P_{\rho} \cdot \nabla \phi_{\rho} d \mu_{\rho}+\lambda\left(G_{\lambda}^{\rho} f_{\rho}, \phi_{\rho}\right)_{\rho}=\left(f_{\rho}, \phi_{\rho}\right)_{\rho} \rightarrow \\
& \int_{B_{\infty}(1)} P_{\lambda}^{*} \cdot \nabla \phi_{\infty} d \mu_{\infty}+\lambda\left(u_{\lambda}^{*}, \phi_{\infty}\right)_{\infty}=\left(f, \phi_{\infty}\right)_{\infty} .
\end{aligned}
$$

Thus it is enough to show that $P_{\lambda}^{*}=\left(q^{i j}\right) \nabla u_{\lambda}^{*}$ on $B_{\infty}$ (1) because it induces $u_{\lambda}^{*}=G_{\lambda} f$.

Second step:

We first take $\chi^{k}(y)$ (see 4.1) such that $\mathcal{M}\left(\chi^{k}\right)=0$ and we define

$$
w_{\rho}(x)=x_{k}-\frac{1}{\rho} \chi^{k}(\rho x)
$$

for every $k=1, \ldots, d_{1}$. Then

$$
w_{\rho} \rightarrow x_{k} \text { strongly in } \mathcal{L}^{2},
$$

and by construction of $\chi^{k}$ (see 4.1) we have

$$
-\partial_{i}\left(\operatorname{det}\left(g_{\rho}\right)^{1 / 2} g_{\rho}^{i j} \partial_{j} w_{\rho}\right)=0 \text { on } B_{\rho}(1) .
$$

We multiply this equation by a test function $\phi \in V_{\rho}$ and after an integration we get

$$
\int_{B_{\rho}(1)} g_{\rho}^{i j} \partial_{j} w_{\rho} \partial_{i} \phi d \mu_{\rho}=0 .
$$


Let $\varphi \in C_{0}^{\infty}\left(B_{\infty}(1)\right)$ (notice that for $\rho$ large enough the support of $\varphi$ will be in $\left.B_{\rho}(1)\right)$ and $\phi=\varphi w_{\rho}$ which we put into Equation (6) and into Equation (13) we put $\phi=\varphi u_{\rho}$ (see (8)), and then we subtract the results:

$$
\begin{aligned}
& \int_{B_{\rho}(1)} g_{\rho}^{i j}\left(\partial_{j} u_{\rho} \partial_{i} \varphi w_{\rho}-\partial_{j} w_{\rho} \partial_{i} \varphi u_{\rho}\right) d \mu_{\rho} \\
& =\int_{B_{\rho}(1)} f_{\rho} w_{\rho} \varphi d \mu_{\rho}-\lambda \int_{B_{\rho}(1)} \varphi u_{\rho} w_{\rho} d \mu_{\rho} .
\end{aligned}
$$

Now let $\rho \rightarrow \infty$ in (14), all terms converge because they are product of one strongly converging net and one weakly converging net in $\mathcal{L}^{2}$. More precisely:

- $P_{\rho}$ defined $P_{\rho, i}=g_{\rho}^{i j} \partial_{j} u_{\rho}$ weakly converges to $P_{\lambda}^{*}$ in $\mathcal{L}^{2}$ following (9).

- $\partial_{i} \varphi w_{\rho}$ strongly converges to $\partial_{i} \varphi x_{k}$ in $\mathcal{L}^{2}$ from (11).

- $g_{\rho}^{i j} \partial_{i} w_{\rho}$ is $D_{f} / \rho$-periodic and weakly converges in $\mathcal{L}^{2}$ towards its mean value

$$
q^{j k}=\mathcal{M}\left(g^{i j}(y)\left(\delta_{i k}-\partial_{i} \chi^{k}(y)\right)\right) .
$$

- $\partial_{j} \varphi u_{\rho}$ strongly converges to $\partial_{j} \varphi u_{\lambda}^{*}$ by (8), because $\varphi$ has compact support.

- Now for the right side, $w_{\rho}$ strongly converges as $u_{\rho}$ does and $f_{\rho}$ weakly converges to $f$.

To summarise (14) converges to (we write $P_{\lambda, i}^{*}$ the coordinates of $P_{\lambda}^{*}$ )

$$
\begin{aligned}
& \int_{B_{\infty}(1)}\left(P_{\lambda, j}^{*} x_{k}-q^{j k} u_{\lambda}^{*}\right) \partial_{j} \varphi d \mu_{\infty} \\
& =\int_{B_{\infty}(1)} f x_{k} \varphi d \mu_{\infty}-\lambda \int_{B_{\infty}(1)} \varphi u_{\lambda}^{*} x_{k} d \mu_{\infty}
\end{aligned}
$$

furthermore if we put into Equation (9), $\phi_{\infty}=\varphi x_{k}$ it gives

$$
\int_{B_{\infty}(1)} f x_{k} \varphi d \mu_{\infty}-\lambda \int_{B_{\infty}(1)} \varphi u_{\lambda}^{*} x_{k} d \mu_{\infty}=\int_{B_{\infty}(1)} P_{\lambda, j}^{*} \partial_{j}\left(\varphi x_{k}\right) d \mu_{\infty}
$$

and by mixing (15) and (16) we get for every $\varphi \in \mathcal{C}_{c}^{\infty}\left(B_{\infty}(1)\right)$ the following equality:

$$
\int_{B_{\infty}(1)}\left(P_{\lambda, j}^{*} x_{k}-q^{j k} u^{*}\right) \partial_{j} \varphi d \mu_{\infty}=\int_{B_{\infty}(1)} P_{\lambda, j}^{*} \partial_{j}\left(\varphi x_{k}\right) d \mu_{\infty}
$$

which in terms of distribution can be translated into:

$$
-\sum_{j=1}^{d_{1}} \partial_{j}\left(P_{\lambda, j}^{*} x_{k}-q^{j k} u_{\lambda}^{*}\right)=-\sum_{j=1}^{d_{1}} \partial_{j} P_{j}^{*} x_{k} \Longleftrightarrow P_{\lambda, k}^{*}=\sum_{j=1}^{d_{1}} q^{j k} \partial_{j} u_{\lambda}^{*}
$$

which allow us to conclude that $u_{\lambda}^{*}=G_{\lambda} f$.

It is now easy to finish the proof of Theorem 1, it comes from Theorem 25 and Theorem 21. 


\section{5. $\Gamma$-convergence of quadratic forms.}

5.1. $\Gamma$ and Mosco-convergence of quadratic forms. We are now going to give a definition of $\Gamma$-convergence adapted to our problem.

Definition 26 ( $\Gamma$-convergence). We say that a net $\left\{F_{\alpha}: L_{\alpha}^{2} \rightarrow \overline{\mathbb{R}}\right\}_{\alpha \in \mathcal{A}}$ of functions $\Gamma$-converges to $F: L_{\infty}^{2} \rightarrow \overline{\mathbb{R}}$ if and only if the following assertions are satisfied:

(F1) For any net $\left(u_{\alpha}\right)_{\alpha \in \mathcal{A}} \in L_{\alpha}^{2}$ strongly converging to $u \in L_{\infty}^{2}$ in $\mathcal{L}^{2}$ we have

$$
F(u) \leq \liminf _{\alpha} F_{\alpha}\left(u_{\alpha}\right) .
$$

(F2) For every $u \in L_{\infty}^{2}$ there is a net $\left(u_{\alpha}\right)_{\alpha \in \mathcal{A}} \in L_{\alpha}^{2}$ strongly converging to $u$ in $\mathcal{L}^{2}$ such that

$$
F(u)=\lim _{\alpha} F_{\alpha}\left(u_{\alpha}\right) .
$$

Remark. This is slightly different from Definition 6, which is the usual one. By taking $F_{\alpha}$ infinite outside of $L_{\alpha}^{2}$ in $\mathcal{L}^{2}$ we get back (in some way) the usual definition (see the introduction of [Mas93]).

Let us summarise some properties satisfied by this convergence.

\section{Lemma 27.}

(a) Let $\left\{F_{\alpha}: L_{\alpha}^{2} \rightarrow \overline{\mathbb{R}}\right\}_{\alpha \in \mathcal{A}}$ be a net of functions $\Gamma$-converging to a function $F: L_{\infty}^{2} \rightarrow \overline{\mathbb{R}}$, then $F$ is lower semi-continuous.

(b) Let $\left(\mathcal{E}_{\alpha}\right)_{\alpha \in \mathcal{A}}$ be a net of quadratic forms $\mathcal{E}_{\alpha}$ on $L_{\alpha}^{2} \Gamma$-converging to a function $F: L_{\infty}^{2} \rightarrow \overline{\mathbb{R}}$, then $F$ can be identified with a quadratic form on $L_{\infty}^{2}$.

There is also the following result, concerning compactness:

Theorem 28. From every net $\left(\mathcal{E}_{\alpha}\right)_{\alpha \in \mathcal{A}}$ of quadratic forms $\mathcal{E}_{\alpha}$ on $L_{\alpha}^{2}$ we can extract a $\Gamma$-converging subnet, whose limit is a quadratic form on $L_{\infty}^{2}$.

Remark. This theorem is true for a wider variety of functions, with some restrictions on $\left\{L_{\alpha}^{2}\right\}_{\nu \in \mathcal{A}}$. Of course the limit in that case is not always a quadratic form. Here it is Lemma 27 which gives information on the limit.

Definition 29 (Mosco topology). We say that a net $\left(\mathcal{E}_{\alpha}\right)_{\alpha \in \mathcal{A}}$ of quadratic forms $\mathcal{E}_{\alpha}$ on $L_{\alpha}^{2}$ Mosco-converges to the quadratic form $\mathcal{E}$ on $L_{\infty}^{2}$ if condition (F2) of Definition 26 and (F1') are satisfied:

$\left(\mathrm{F} 1^{\prime}\right)$ For any $\left(u_{\alpha}\right)_{\alpha \in \mathcal{A}}, u_{\alpha} \in L_{\alpha}^{2}$ weakly converging net to $u \in L_{\infty}^{2}$ in $\mathcal{L}^{2}$ we have

$$
\mathcal{E}(u) \leq \liminf _{\alpha} \mathcal{E}_{\alpha}\left(u_{\alpha}\right)
$$

The induced topology is called the Mosco topology.

It is obvious that the Mosco-convergence induces the $\Gamma$-convergence, thus this topology is stronger. Let us now define one last convergence: 
Definition 30 (Compact $\Gamma$-convergence). We say that a net $\left(\mathcal{E}_{\alpha}\right)_{\alpha \in \mathcal{A}} \Gamma$ converges compactly to $\mathcal{E}$ if $\mathcal{E}_{a} \rightarrow \mathcal{E}$ in the Mosco topology an if $\left(\mathcal{E}_{a}\right)_{a \in \mathcal{A}}$ is asymptotically compact.

Let us show precisely how the Mosco and the $\Gamma$ topologies are linked:

Lemma 31. Let us suppose $\left(\mathcal{E}_{\alpha}\right)_{\alpha \in \mathcal{A}}$ asymptotically compact then $\left(\mathcal{E}_{\alpha}\right)_{\alpha \in \mathcal{A}}$ $\Gamma$-converges to $\mathcal{E}$ is and only if $\left(\mathcal{E}_{\alpha}\right)_{\alpha \in \mathcal{A}}$ Mosco-converges to $\mathcal{E}$.

Proof. We just need to show that the $\Gamma$-convergence implies the condition $\left(\mathrm{F} 1^{\prime}\right)$ from Definition 29. We proceed ad absurdum and suppose that there is a weakly converging net $\left(u_{\alpha}\right)$ such that $\liminf _{\alpha} \mathcal{E}_{\alpha}\left(u_{\alpha}\right)<\mathcal{E}(u)$. Taking a subnet if necessarily we can suppose $\lim \mathcal{E}_{\alpha}\left(u_{\alpha}\right)<\mathcal{E}(u)$ thus we also have $\lim \sup _{\alpha} \mathcal{E}_{\alpha}\left(u_{\alpha}\right)+\left\|u_{\alpha}\right\|_{\alpha}^{2}<+\infty$. The asymptotic compactness is obviously inherited by a subnet thus we can extract a strongly converging subnet $u_{\alpha(\beta)}$. The $\Gamma$-convergence being also inherited by a subnet of $\mathcal{E}_{\alpha}$ we finally get

$$
\lim \mathcal{E}_{\alpha}\left(u_{\alpha}\right)=\lim _{\beta} \mathcal{E}_{\alpha(\beta)}\left(u_{\alpha(\beta)}\right) \geq \mathcal{E}(u)
$$

which is absurd.

5.2. $\Gamma$-convergence and spectral structures. The following theorem explains how the convergence of spectral structures and the Mosco-convergence are related:

Theorem 32. Let $\left(\Sigma_{\alpha}\right)$ be a net of spectral structures on $\left(L_{\alpha}^{2}\right)$ and $\Sigma$ a spectral structure on $L_{\infty}^{2}$ then $\Sigma_{\alpha} \longrightarrow \Sigma$ strongly (resp. compactly) if and only if $\mathcal{E}_{\alpha}$ Mosco-converges (resp. $\Gamma$-converges compactly) to $\mathcal{E}$.

Proof. We are going to prove the equivalence between the strong (resp. compact) convergence of resolvents and the Mosco-convergence (resp. compact $\Gamma$-convergence) of the energies.

Let us begin by assuming the Mosco-convergence of the net $\left(\mathcal{E}_{\alpha}\right)$. We need to show that for every $z \in L_{\infty}^{2}$ and any net $\left(z_{\alpha}\right)$ strongly converging to $z$ the net $u_{\alpha}=-R_{\lambda}^{\alpha} z_{\alpha}$ strongly converges to $u=-R_{\lambda} z$. First let us notice that the vector $u$ is the unique minimiser of

$$
v \mapsto \mathcal{E}(v)-\lambda\|v\|_{\infty}^{2}-2\langle z, v\rangle_{\infty}
$$

we can characterise the same way $u_{\alpha}$ for every $\alpha$.

As an operator of $L_{\alpha}^{2}, R_{\lambda}^{\alpha}$ is bounded by $-\lambda^{-1}$. Thus the net $\left(u_{\alpha}\right)$ is bounded and we can extract a weakly converging subnet, still written $\left(u_{\alpha}\right)$, with limit $\widetilde{u}$. Now from condition (F2) for every $v \in L_{\infty}^{2}$ there is a net strongly converging to it such that $\lim _{\alpha} \mathcal{E}_{\alpha}\left(v_{\alpha}\right)=\mathcal{E}(v)$. But for every $\alpha$

$$
\mathcal{E}_{\alpha}\left(u_{\alpha}\right)-\lambda\left\|u_{\alpha}\right\|_{\alpha}^{2}-2\left\langle z_{\alpha}, u_{\alpha}\right\rangle_{\alpha} \leq \mathcal{E}_{\alpha}\left(v_{\alpha}\right)-\lambda\left\|v_{\alpha}\right\|_{\alpha}^{2}-2\left\langle z_{\alpha}, v_{\alpha}\right\rangle_{\alpha}
$$

thus taking the limit in $\alpha \in \mathcal{A}$ we get thanks to condition (F1') of Definition 29 and the fact that for any weakly convergent net $\|\widetilde{u}\|_{\infty} \leq \liminf _{\alpha}\left\|u_{\alpha}\right\|_{\alpha}$ 
(remember that $\lambda<0$ )

$$
\mathcal{E}(\widetilde{u})-\lambda\|\widetilde{u}\|_{\infty}^{2}-2\langle z, \widetilde{u}\rangle_{\infty} \leq \mathcal{E}(v)-\lambda\|v\|_{\infty}^{2}-2\langle z, v\rangle_{\infty}
$$

which implies $\widetilde{u}=-R_{\lambda} z$. Due to $u$ 's unicity, we conclude that $\left(u_{\alpha}\right)$ weakly converges to $u$. Let us prove that $\left\|u_{\alpha}\right\|_{\alpha}$ converges to $\|u\|_{\infty}$. In that aim take a strongly convergent net $v_{\alpha}$ to $v$ such that $\lim _{\alpha} \mathcal{E}_{\alpha}\left(v_{\alpha}\right)=\mathcal{E}(u)$, and take a new look at inequality (17):

$$
\mathcal{E}_{\alpha}\left(u_{\alpha}\right)-\lambda\left\|u_{\alpha}+z_{\alpha} / \lambda\right\|_{\alpha}^{2} \leq \mathcal{E}_{\alpha}\left(v_{\alpha}\right)-\lambda\left\|v_{\alpha}+z_{\alpha} / \lambda\right\|_{\alpha}^{2}
$$

using $\left(\mathrm{F} 1^{\prime}\right)$ once again we find

$$
\mathcal{E}(v)-\lambda \limsup _{\alpha}\left\|u_{\alpha}+z_{\alpha} / \lambda\right\|_{\alpha}^{2} \leq \mathcal{E}(v)-\lambda\|u+z / \lambda\|_{\infty}^{2}
$$

thus $\left\|u_{\alpha}+z_{\alpha} / \lambda\right\|_{\alpha}^{2} \rightarrow\|u+z / \lambda\|_{\infty}^{2}$ which implies the strong convergence of $\left(u_{\alpha}+z_{\alpha} / \lambda\right)_{\alpha}$ and the strong convergence of $\left(z_{\alpha}\right)$ induces the strong convergence of $\left(u_{\alpha}\right)$.

We shall now study the compact $\Gamma$-convergence. Let us take a weakly convergent net $w_{\alpha}$ to $w$ and let $u_{\alpha}=-R_{\lambda}^{\alpha} w_{\alpha}$, then the net $u_{\alpha}$ is still bounded. Swapping $z_{\alpha}$ with $w_{\alpha}$ in (17) we get that $\lim \sup _{\alpha} \mathcal{E}_{\alpha}\left(u_{\alpha}\right)$ is bounded, and thanks to the asymptotic compactness we can extract a strongly convergent subnet with $\widetilde{u}$ its limit. Putting this in (17), with $z_{\alpha}=v_{\alpha}$ where $\left(v_{\alpha}\right)$ a strongly converging net to $v$ we get

$$
\mathcal{E}(\widetilde{u})-\lambda\|\widetilde{u}\|^{2}-2\langle w, \widetilde{u}\rangle \leq \mathcal{E}(v)-\lambda\|v\|^{2}-2\langle w, v\rangle
$$

thus $\widetilde{u}=-R_{\lambda} w$. Once again, thanks to unicity, we conclude that $R_{\lambda}^{\alpha} w_{\alpha}$ strongly converges to $R_{\lambda} w$.

Reciprocally assume that for every $\lambda<0$ the net $R_{\lambda}^{\alpha}$ strongly converges to $R_{\lambda}$. In what follows $\left(u_{\alpha}\right)$ will be a strong convergent net to $u$.

Condition (F1'): Already done, see Proposition 20.

Condition (F2): Extract a subnet $\lambda_{\alpha} \rightarrow-\infty$ such that

$$
\mathcal{E}(u, u) \geq \lim _{\lambda} \lim _{\alpha} a_{\alpha}^{\lambda}\left(u_{\alpha}, u_{\alpha}\right) \geq \lim _{\alpha} a_{\alpha}^{\lambda_{\alpha}}\left(u_{\alpha}, u_{\alpha}\right)
$$

take $w_{\alpha}=\lambda_{\alpha} R_{\lambda_{\alpha}}^{\alpha} u_{\alpha}$ for every $\alpha$ and notice that

$$
\begin{aligned}
a_{\alpha}^{\lambda}\left(u_{\alpha}, u_{\alpha}\right)= & -\lambda\left\langle u_{\alpha}-\lambda R_{\lambda}^{\alpha} u_{\alpha}, u_{\alpha}\right\rangle_{\alpha}-\lambda\left\langle u_{\alpha}-\lambda R_{\lambda}^{\alpha} u_{\alpha},-\lambda R_{\lambda}^{\alpha} u_{\alpha}\right\rangle_{\alpha} \\
& +\lambda\left\langle u_{\alpha}-\lambda R_{\lambda}^{\alpha} u_{\alpha},-\lambda R_{\lambda}^{\alpha} u_{\alpha}\right\rangle_{\alpha} \\
= & -\lambda\left\|u_{\alpha}-\lambda R_{\lambda}^{\alpha} u_{\alpha}\right\|^{2}+\lambda^{2}\left\langle u_{\alpha}-\lambda R_{\lambda}^{\alpha} u_{\alpha},-R_{\lambda}^{\alpha} u_{\alpha}\right\rangle_{\alpha} \\
= & -\lambda\left\|u_{\alpha}-\lambda R_{\lambda}^{\alpha} u_{\alpha}\right\|^{2}+\mathcal{E}_{\alpha}\left(\lambda R_{\lambda}^{\alpha} u_{\alpha}\right)
\end{aligned}
$$

indeed if $a_{\alpha}$ is the bilinear form corresponding to $\mathcal{E}_{\alpha}$ then $R_{\lambda}^{\alpha} u_{\alpha}$ can be seen as the sole element such that

$$
a_{\alpha}\left(-R_{\lambda}^{\alpha} u_{\alpha}, v_{\alpha}\right)-\lambda\left\langle-R_{\lambda}^{\alpha} u_{\alpha}, v_{\alpha}\right\rangle_{\alpha}=\left\langle u_{\alpha}, v_{\alpha}\right\rangle_{\alpha}, \quad \forall v_{\alpha} \in D\left(\mathcal{E}_{\alpha}\right)
$$


hence

$$
a_{\alpha}^{\lambda_{\alpha}}\left(u_{\alpha}, u_{\alpha}\right)=\mathcal{E}_{\alpha}\left(w_{\alpha}, w_{\alpha}\right)-\lambda_{\alpha}\left\|u_{\alpha}-w_{\alpha}\right\|^{2}
$$

which implies $w_{\alpha} \rightarrow u$ strongly in $\mathcal{L}^{2}$ and

$$
\mathcal{E}(u, u) \geq \limsup _{\alpha \rightarrow+\infty} \mathcal{E}_{\alpha}\left(w_{\alpha}, w_{\alpha}\right) .
$$

For the compact convergence case it suffices to prove the asymptotic compactness, but it has already been done in the proof of Proposition 20.

\section{Proof of Theorem 2.}

The convergence of the eigenvalue is given by Theorem 1 . Hence it remains to bound the asymptotic $\lambda_{1}$ (i.e., the limit) and characterise the equality. The proof we propose consists in finding an upper bound of $\lambda_{1}\left(B_{g}(\rho)\right)$ for every $\rho$ using a function depending of the distance from the centre of the ball. We then use the simple convergence of the distances $\left(d_{\rho}\right)$ to the stable norm as seen in Section 2.2 and the measure part of Theorem 10.

Proof. Let $f$ be a continuous function from $\mathbb{R}$ to $\mathbb{R}$ and define

$$
\begin{aligned}
f_{\rho}: B_{\rho}(1) & \rightarrow \mathbb{R} \\
x & \mapsto f\left(d_{\rho}(0, x)\right)
\end{aligned}
$$

and $f_{\infty}(x)=f\left(\|x\|_{\infty}\right)$ on $B_{\infty}(1)$. We want to show that (remember that $\left.\delta_{\rho}(x)=\rho x\right)$

$$
\int f_{\rho} \cdot \chi_{B_{g}(\rho)} \circ \delta_{\rho} d \mu_{\rho} \underset{\rho \rightarrow \infty}{\longrightarrow} \int_{B_{\infty}(1)} f_{\infty} d \mu_{\infty} .
$$

To obtain this we are going to cut the difference in three pieces, i.e.,

$$
\begin{aligned}
& \left|\int f_{\rho} \cdot \chi_{B_{g}(\rho)} \circ \delta_{\rho} d \mu_{\rho}-\int_{B_{\infty}(1)} f_{\infty} d \mu_{\infty}\right| \\
& \leq\left|\int f_{\rho} \cdot\left(\chi_{B_{g}(\rho)} \circ \delta_{\rho}-\chi_{B_{\infty}(1)}\right) d \mu_{\rho}\right| \\
& \quad+\left|\int_{B_{\infty}(1)}\left(f_{\rho}-f_{\infty}\right) d \mu_{\rho}\right| \\
& \quad+\left|\int_{B_{\infty}(1)} f_{\infty} d \mu_{\rho}-\int_{B_{\infty}(1)} f_{\infty} d \mu_{\infty}\right| .
\end{aligned}
$$

Now it suffices to notice that:

1) Part (19) goes to 0 because inside we have the product of $\chi_{B_{g}(\rho)} \circ$ $\delta_{\rho}-\chi_{B_{\infty}(1)}$, which is easily seen to simply converge to 0 thanks to Corollary 9, with bounded terms compactly supported.

2) Same reason for (20) because $f_{\rho}-f_{\infty}$ simply converges to 0 . 
3) Finally the convergence to 0 of (21) is due once again to the measure part of Theorem 10.

As a conclusion we have (18). Injecting now $f_{\rho}$ into the Raleigh's quotient we get:

$$
\rho^{2} \lambda_{g}\left(B_{g}(\rho)\right) \leq \frac{\int\left(\left(f^{\prime}\right)_{\rho}\right)^{2} \cdot \chi_{B_{g}(\rho)} \circ \delta_{\rho} d \mu_{\rho}}{\int\left(f_{\rho}\right)^{2} \cdot \chi_{B_{g}(\rho)} \circ \delta_{\rho} d \mu_{\rho}} .
$$

We apply the limit (18) to obtain

$$
\limsup _{\rho \rightarrow \infty} \rho^{2} \lambda_{g}\left(B_{g}(\rho)\right) \leq \frac{\int_{B_{\infty}(1)}\left(\left(f^{\prime}\right)_{\infty}\right)^{2} d \mu_{\infty}}{\int_{B_{\infty}(1)} f_{\infty}^{2} d \mu_{\infty}}
$$

and now taking for $f$ the right function (i.e., the solution of the differential equation $\left.f^{\prime \prime}+\frac{n-1}{x} f^{\prime}(x)+\lambda_{e, n} f=0\right)$ we can conclude.

Let us now study the equality case. Take again the function $f$ which gives the eigenfunction of the Euclidean Laplacian on the Euclidean unit ball (i.e., the solution of $f^{\prime \prime}+\frac{n-1}{x} f^{\prime}(x)+\lambda_{e, n} f=0$ ) and normalise it. The $\Gamma$-convergence theory allows to say, taking $\mathcal{E}_{\rho}$ and $\mathcal{E}_{\infty}$ as the energies of $\Delta_{\rho}$ and $\Delta_{\infty}$ on the balls $B_{\rho}(1)$ and $B_{\infty}(1)$ respectively for the adapted measures and thanks to Proposition 20 and Theorem 1

$$
\mathcal{E}_{\infty}\left(f_{\infty}\right) \leq \liminf _{\rho \rightarrow \infty} \mathcal{E}_{\rho}\left(f_{\rho}\right) \leq \limsup _{\rho \rightarrow \infty} \mathcal{E}_{\rho}\left(f_{\rho}\right) \leq \lambda_{e, n} .
$$

Now from the equality assumption we have

$$
\lambda_{e, n} \leq \mathcal{E}_{\infty}\left(f_{\infty}\right),
$$

thus (22) and (23) imply equality which in turn imply that $f_{\infty}$ is an eigenfunction for the first eigenvalue . Hence $f_{\infty}$ is smooth (at least in a neighbourhood of zero).

Now from the study of Bessel's function (see [Bow58], $§ 103-\S 105)$ we see that taking $p=(n-2) / 2$ we have $f(x)=x^{-p} J_{p}\left(\sqrt{\lambda_{e}} x\right)$ with $J_{p}$ an analytic function defined by (see F. Bowman [Bow58] 884 )

$$
J_{p}(x)=\frac{x^{p}}{2^{p} \Gamma(p+1)}\left(1-\frac{x^{2}}{2 \cdot 2 n+2}+\frac{x^{4}}{2 \cdot 4 \cdot 2 n+2 \cdot 2 n+4}+\cdots\right)
$$

thus $f$ has the following shape:

$$
f(x)=\frac{\lambda_{e}^{p}}{2^{p} \Gamma(p+1)}\left(1-\frac{x^{2} \lambda_{e}}{2 \cdot 2 n+2}+\frac{x^{4} \lambda_{e}^{2}}{2 \cdot 4 \cdot 2 n+2 \cdot 2 n+4}+\cdots\right)
$$

in other words $f$ has the following asymptotic expansion: $1+\alpha_{1} x^{2}+\alpha_{2} x^{4}+\cdot \cdot$ (up to a multiplicative constant). Now notice that the function $1+\alpha_{1} x+$ $\alpha_{2} x^{2}+\cdots$ admits an inverse $g \in C^{\infty}$ in a neighbourhood of zero, which 
implies that $g \circ f_{\infty}(x)=c s t \cdot\|x\|_{\infty}^{2}$ is $C^{2}$ in a neighbourhood of zero, thus the stable norm comes from a scalar products, which means that it is Euclidean.

In fact we have some more informations. Indeed in order for $f_{\infty}$ to be an eigenfunction, the norme of the differential of the stable norm with respect to the Albanese metric (the scalar product giving the Laplacian $\Delta_{\infty}$ ) must be almost everywhere equal to one (a simple computation using the fact that the stable norm is Euclidean and the Cauchy-Schwartz inequality). Which implies that the unit ball of the Albanese metric must be inside the unit ball of the stable norm. Now the maximum principle and the monotony with respect to inclusion of the eigenvalues implies that equality holds if and only if the stable norm and the Albanese metric coincides. The stable norm being the Albanese metric we can now use Theorem 33 to conclude.

Theorem 33. Let $\left(\mathbb{T}^{n}, g\right)$ be a torus, its stable norm coincides with the Albanese metric if and only if the torus is flat.

Proof. Let us take a base $\eta_{1}, \ldots, \eta_{n}$ of Harmonic one forms, any function $\alpha$ and any 2 -form $\beta$. We shall write $(\cdot, \cdot)_{g}$ the pointwise scalar product induced by $g$ on forms $\left(\|\cdot\|_{g}\right.$ the associated norm) and $\langle\cdot, \cdot\rangle_{g}$ the integral scalar product normalized by the volume. Then by Hodge's theorem

$$
\left\|\eta_{i}\right\|_{\infty}^{2}=\inf _{\alpha, \beta} \sup _{x \in \mathbb{T}^{n}}\left\|\eta_{i}\right\|_{g}^{2}+\|d \alpha\|_{g}^{2}+\|\delta \beta\|_{g}^{2} \geq \sup _{x \in \mathbb{T}^{n}}\left\|\eta_{i}\right\|_{g}^{2}
$$

and

$$
\left\langle\eta_{i}, \eta_{i}\right\rangle_{g}=\frac{1}{\operatorname{Vol}_{g}\left(\mathbb{T}^{n}\right)} \int_{\mathbb{T}^{n}}\left\|\eta_{i}\right\|_{g}^{2} d \mathrm{vol}_{g} \leq \sup _{x \in \mathbb{T}^{n}}\left\|\eta_{i}\right\|_{g}^{2}
$$

the case of equality implies that $\left\langle\eta_{i}, \eta_{i}\right\rangle_{g}=\left(\eta_{i}, \eta_{i}\right)_{g}(x)$ for all $x \in \mathbb{T}^{n}$. Now it suffices to see that the metric $g$ can be written in the following way:

$$
\sum_{i, j} \lambda_{i j} \eta_{i} \circ \eta_{j}=g
$$

where $\eta_{i} \circ \eta_{j}=1 / 2\left(\eta_{i} \otimes \eta_{j}+\eta_{j} \otimes \eta_{i}\right)$ and $\Lambda=\left(\lambda_{i j}\right)$ is the matrice such that $\Lambda^{-1}=\left(\left\langle\eta_{i}, \eta_{j}\right\rangle_{g}\right)$. Now taking local $f_{i}$ such that $d f_{i}=\eta_{i}$ then the function $F(x)=\left(f_{1}(x), \ldots, f_{n}(x)\right)$ is an isometry between an open set of $\mathbb{T}^{n}$ and an eucliean space, thus the torus is flat.

\section{Related topics.}

In that section we come back to the asymptotic volume, proving in the meantime a generalised Faber-Krahn inequality. Then we explain what can be deduced from our work for the heat kernel and how it is related to other's work. We finally state how Theorem 1 passes to graded nilmanifolds. 


\subsection{Asymptotic volume of tori.}

7.1.1. Generalised Faber-Krahn inequality. We need some more definitions.

Definition 34. For a rectifiable submanifold $N$ of $\mathbb{R}^{n}$ (we can think of it of finite adapted Hausdorff measure) we will write $I(N)$ the associated integral current. For an integral current $C, M(C)$ will be its mass as defined par H. Federer (see [Fed69] for example).

Definition 35. Let $\mathbb{R}^{n}$, with the norm $\|\cdot\|\left(\|\cdot\|_{*}\right.$ will be the dual norm), we define

$$
\lambda_{1}(\Omega,\|\cdot\|)=\inf _{f} \frac{\int_{\Omega}\|d f\|_{*}^{2} d \mu}{\int_{\Omega} f^{2} d \mu}
$$

where $\mu$ is the Lebesgue measure on $\mathbb{R}^{n}$, and the infimum is taken over all Lipschitz functions vanishing on the border.

The following lemma holds:

Lemma 36 (Faber-Krahn inequality for norms). Let $D$ be a domain of $\mathbb{R}^{n}$, with the norm $\|\cdot\|$ and a measure $\mu$ invariant by translation. Let $D^{*}$ be the norm's ball with same measure as $D$, then

$$
\lambda_{1}\left(D^{*},\|\cdot\|\right) \leq \lambda_{1}(D,\|\cdot\|)
$$

the equality case implying that $D$ is a norm's ball.

Proof. We need two ingredients for this proof. The first is an isoperimetric inequality, which is given by a result of Brunn (see a proof by M. Gromov in [MS86]). The second is a co-area formula, which can be found in Federer [Fed69] p. 438.

More specifically, let us write $G_{t}=\{x|| f(x) \mid=t\}$ then on one side we have

$$
\int_{\Omega} h \alpha \wedge d f=\int_{0}^{\sup f} \int_{G_{t}} h \alpha_{\mid G_{t}} d t=\int_{0}^{\sup f} I_{|f|=t}(h \alpha) d t
$$

and on the other

$$
\int_{\Omega}\|d f\|_{*} d \mu=\int_{0}^{\sup f} M\left(I_{|f|=t}\right) d t
$$

(see P. Pansu [Pan99]) where $d \mu$ is the translation invariant volume form on $\mathbb{R}^{n}$ such that the norm's ball of radius one has measure 1 .

Take $\alpha=\frac{1}{|d f|^{2}} * d f$ where $*$ is the Hodge operator on differential forms over $\mathbb{R}^{n}$ then we get

$$
\int_{\Omega} h d \mu=\int_{0}^{\sup f} \int_{G_{t}} h \alpha_{\mid G_{t}} d t
$$


Now take a look at the same equality on $\Omega_{t}=\{x|| f(x) \mid>t\}$ i.e.,

$$
\int_{\Omega_{t}} h d \mu=\int_{t}^{\sup f} \int_{G_{t}} h \alpha_{\mid G_{t}} d t=\int_{t}^{\sup f} I_{|f|=t}(h \alpha) d t .
$$

Differentiating each member of equality (26) we get almost everywhere the following equality:

$$
\int_{G_{t}} h \alpha_{\mid G_{t}}=I_{|f|=t}(h \alpha) .
$$

Taking into account (27) and (25) we obtain

$$
\int_{G_{t}}\|d f\|_{*} \alpha_{\mid G_{t}}=M\left(I_{|f|=t}\right) .
$$

Applying the Cauchy-Schwartz inequality to the left side of (28) and making the appropriate identification thanks to (27) we finally have

$$
\frac{M\left(I_{|f|=t}\right)^{2}}{I_{|f|=t}(\alpha)} \leq I_{|f|=t}\left(\left(\|d f\|_{*}\right)^{2} \alpha\right) .
$$

The function $f^{*}$ associated to $f$ by symmetrisation is Lipschitz. Thus it satisfies a similar co-area formula. Hence we have for almost all $t$

$$
I_{|f|=t}(\alpha)=-\frac{d}{d t} \operatorname{Vol}\left(\Omega_{t}\right)=-\frac{d}{d t} \operatorname{Vol}\left(\Omega_{t}^{*}\right)=I_{\left|f^{*}\right|=t}\left(\alpha^{*}\right) .
$$

Now using Brunn's isoperimetric inequality (see [MS86]) we have

$$
M\left(I_{\left|f^{*}\right|=t}\right) \leq M\left(I_{|f|=t}\right) .
$$

Injecting (30) and (31) in (29) and noticing that $\left\|d f^{*}\right\|_{*}$ is constant on $\{|f|=$ $t$ \}, which implies that the equivalent of (29) for $f^{*}$ is an equality we get (for almost all $t$ )

$$
\begin{aligned}
I_{\left|f^{*}\right|=t}\left(\left(\left\|d f^{*}\right\|_{*}\right)^{2} \alpha^{*}\right)=\frac{M\left(I_{\left|f^{*}\right|=t}\right)^{2}}{I_{\left|f^{*}\right|=t}\left(\alpha^{*}\right)} & \leq \frac{M\left(I_{|f|=t}\right)^{2}}{I_{|f|=t}(\alpha)} \\
& \leq I_{|f|=t}\left(\left(\|d f\|_{*}\right)^{2} \alpha\right) .
\end{aligned}
$$

Now we sum the extremal terms of (32) to obtain the desired inequality:

$$
\int_{\Omega^{*}}\left(\left\|d f^{*}\right\|_{*}\right)^{2} d v \leq \int_{\Omega}\left(\|d f\|_{*}\right)^{2} d v
$$

which allows us to conclude the proof because

$$
\int_{\Omega^{*}}\left(f^{*}\right)^{2} d v=\int_{\Omega}(f)^{2} d v .
$$

For the equality case, it suffices to see that it implies the equality case in Brunn's isoperimetric inequality to conclude.

Let us notice that this lemma immediately implies: 
Corollary 37. Let $D_{1}$ be the unit ball of the norm $\|\cdot\|$. Then

$$
\lambda_{1}\left(D_{1}\right)=\lambda_{e, n} .
$$

Thus

$$
\lambda_{e, n}\left(\frac{\mu\left(D_{1}\right)}{\mu(D)}\right)^{\frac{2}{n}} \leq \lambda_{1}(D,\|\cdot\|)
$$

where $\mu$ is a Haar measure on $\mathbb{R}^{n}$.

Proof. The symmetrisation from the previous theorem shows that the minimum of the Rayleigh's quotient is obtained with functions depending on the distance from the centre of the ball. Hence we are led to the same calculations as in the Euclidean case.

7.1.2. Lower bound for the asymptotic volume. We are now going to apply the generalised Faber-Krahn inequality to $\lambda_{\infty}$. With that aim in mind let us notice that $\lambda_{\infty}\left(B_{\infty}(1)\right)=\lambda_{1}\left(B_{\infty}(1),\|\cdot\|_{2}^{*}\right)$ with the dual norm of $\|\cdot\|_{2}^{*}$ defined by

$$
\|\xi\|_{2}=\sum_{i j} q^{i j} \xi_{i} \xi_{j}
$$

and let us write $B_{\mathrm{Al}}$ the unit ball of $\|\cdot\|_{2}^{*}$. We now can apply the inequality of Lemma 36 and more precisely its Corollary 37:

$$
\lambda_{\infty}\left(B_{\infty}(1)\right) \geq\left(\frac{\mu\left(B_{\mathrm{Al}}\right)}{\mu\left(B_{\infty}(1)\right)}\right)^{2 / n} \lambda_{e, n}
$$

where $\mu$ is any Haar measure. Now applying Theorem 2 we get

$$
\left(\frac{\mu\left(B_{\mathrm{Al}}\right)}{\mu\left(B_{\infty}(1)\right)}\right)^{2 / n} \lambda_{e, n} \leq \lambda_{e, n} .
$$

We finally get the following proposition taking in (33) the Haar measure such that the measure of $B_{\infty}(1)$ is the asymptotic volume (i.e., the measure $\left.\mu_{\infty}\right)$ and transforming the other term in order to make the Albanese torus's volume appear.

Proposition 3. Let $\left(\mathbb{T}^{n}, g\right)$ be a Riemannian torus, $B_{g}(\rho)$ the geodesic balls of radius $\rho$ centred on a fixed point and $\operatorname{Vol}_{g}\left(B_{g}(\rho)\right)$ their Riemannian volume induced on the universal cover, writing

$$
\operatorname{Asvol}(g)=\lim _{\rho \rightarrow \infty} \frac{\operatorname{Vol}_{g}\left(B_{g}(\rho)\right)}{\rho^{n}}
$$

then:

(1) $\operatorname{Asvol}(g) \geq \frac{\operatorname{Vol}_{g}\left(\mathbb{T}^{n}\right)}{\operatorname{Vol}_{\mathrm{Al}}\left(\mathbb{T}^{n}\right)} \omega_{n}$.

(2) In case of equality, the torus is flat.

Here $\omega_{n}$ is the unit Euclidean ball's Euclidean volume. 
Proof. There remains the equality case to be proved, which can be obtained using either the equality case of the Faber-Krahn inequality, which says that $B_{\infty}(1)$ is an ellipsoid either the equality case of Theorem 2 and then we conclude by using Theorem 33 .

We still have two remarks concerning this proposition, the first one is included in the following corollary:

Corollary 4. For $n=2$ we have:

(1) $\operatorname{Asvol}(g) \geq \pi=\omega_{2}$.

(2) In case of equality, the torus is flat.

In other words we obtain the theorem of D. Burago and S. Ivanov on the asymptotic volume of tori in the 2 dimensional case (see [BI95]). The second remark is that we can not do better this way. See [Ver01] Part Three for more details.

7.2. Long time asymptotics of the heat kernel. Let $\left(\mathbb{T}^{n}, g\right)$ be a torus and $\left(\mathbb{R}^{n}, \widetilde{g}\right)$ its universal cover with the lifted metric. We remind the reader that $g_{\rho}=\left(1 / \rho^{2}\right) \delta_{\rho}^{*} \widetilde{g}$ are the rescaled metrics and $\Delta_{\rho}$ their Laplacian, here it will be on $\mathbb{R}^{n}$.

We are going to study from the homogenisation point of view the long time asymptotic behaviour of the heat kernel i.e., we are interested in the behaviour as $t$ goes to infinity of a solution $u(t, x)$ of the following problem:

$$
\left\{\begin{array}{l}
\frac{\partial u}{\partial t}+\Delta u=0 \\
u(0, x)=u_{0}(x) .
\end{array}\right.
$$

For a probabilistic insight one could see M. Kotani and T. Sunada [KS00].

Let us introduce the rescaled functions

$$
u_{\rho}(t, x)=\rho^{n} u\left(\rho^{2} t, \delta_{\rho} x\right), \rho>0 .
$$

It is straightforward that (see. Section 2.4) $u$ is a solution of (34) if and only if $u_{\rho}$ is a solution of

$$
\left\{\begin{array}{l}
\left.\frac{\partial u_{\rho}}{\partial t}+\Delta_{\rho} u_{\rho}=0 \quad \text { in }\right] 0,+\infty\left[\times \mathbb{R}^{n}\right. \\
u_{\rho}(0, x)=\rho^{n} u_{0}\left(\delta_{\rho} x\right),
\end{array}\right.
$$

hence studying $u(t, \cdot)$ as $t$ goes to infinity is the same as studying $u_{\rho}(1, \cdot)$ as $\rho \rightarrow \infty$. In other words we are once again lead to the study of the spectral structures $\left(\Delta_{\rho}\right)$ on $\mathbb{R}^{n}$. We have:

Theorem 38. The net of resolvents $\left(R_{\lambda}^{\rho}\right)$ weakly converges to the resolvent $\left(R_{\lambda}^{\infty}\right)$ of $\Delta_{\infty}$ in $L^{2}\left(\mathbb{R}^{n}\right)$. 
Remark. The proof is the same as 25 . In fact in that case we would rather talk of $G$-convergence. We now can apply the theorems from Chapter III of [ZKON79], more precisely Theorems 4 and 6 .

Theorem 39 ([ZKON79] p. 136). The fundamental solution $k(t, x, y)$ of (34) has he following asymptotic expansion:

$$
k(t, x, y)=k_{\infty}(t, x, y)+t^{-\frac{n}{2}} \theta(t, x, y)
$$

where $k_{\infty}(t, x, y)$ is fundamental solution of

$$
\left.\frac{\partial u_{\infty}}{\partial t}+\Delta_{\infty} u_{\infty}=0 \text { in }\right] 0,+\infty\left[\times \mathbb{R}^{n}\right.
$$

and $\theta(t, x, y) \rightarrow 0$ uniformly as $t \rightarrow \infty$ on $|x|^{2}+|y|^{2} \leq$ at, for any fixed constant $a>0$.

Remark. This is slightly weaker than Theorem 1 of M. Kotani and T. Sunada in [KS00].

Theorem 40 ([ZKON79] p. 138). Let $u_{0} \in L^{1}\left(\mathbb{R}^{n}\right) \cap L^{\infty}\left(\mathbb{R}^{n}\right)$. Then $u(t, x)$ the solution of (34) has the following asymptotic expansion:

$$
u(t, x)=c_{0}(4 \pi t)^{-\frac{n}{2}} \int_{\mathbb{R}^{n}} u_{0}(y) d y+t^{-\frac{n}{2}} \theta(t, x)
$$

where $\theta(t, x)$ converges uniformly to 0 for $|x|<R$ where $R$ is a positive constant and $c_{0}$ is the determinant of the matrix associated to $\Delta_{\infty}$.

That last claim can be made precise by:

Theorem 41 (Duro, Zuazua [DZ00]). Let $u_{0} \in L^{1}\left(\mathbb{R}^{n}\right)$. The sole solution of (34) satisfies for every $p \in[1,+\infty[$ :

$$
t^{n / 2(1-1 / p)}\left\|u(t)-u_{\infty}(t)\right\|_{p} \rightarrow 0, \text { as } t \rightarrow+\infty
$$

where $u_{\infty}$ is the unique solution of the homogenised problem (36). For $n=1$ and $n=2$ (37) is also true for $p=\infty$.

7.3. The macroscopical sound of graded nilmanifolds. In this part we want to emphasise the fact that Theorem 1 is still true for graded nilmanifolds, at least for the Dirichlet case, but it involves some sub-Riemannian geometry. We just give the statement. The details are to be found in [Ver01] Chapter Two.

Theorem 42. Let $\left(M^{n}, g\right)$ be graded nilmanifold, $B_{g}(\rho)$ the induced Riemannian ball of radius $\rho$ on its universal cover and $\lambda_{i}\left(B_{g}(\rho)\right)$ the $i^{\text {th }}$ eigenvalue of the Laplacian on $B_{g}(\rho)$ for the Dirichlet problem.

Then there exists an hypoelliptic operator $\Delta_{\infty}$ (the Kohn Laplacian of a left invariant metric), whose $i^{\text {th }}$ eigenvalue for the Dirichlet problem on the stable ball is $\lambda_{i}^{\infty}$ and such that

$$
\lim _{\rho \rightarrow \infty} \rho^{2} \lambda_{i}\left(B_{g}(\rho)\right)=\lambda_{i}^{\infty} .
$$


Here the stable ball is the metric ball given by the Carnot-Caratheodory distance found in [Pan82] and arising from the stable norm.

\section{Proof of Theorem 21.}

Let $\left(\Sigma_{\alpha}\right)$ be a net of spectral structures and let us focus on the spectra. For a fixed operator $\sigma(\cdot)$ will be its spectrum. Let us begin with the case of strong convergence.

Proposition 43. If $\Sigma_{\alpha} \rightarrow \Sigma$ strongly, then for any $\lambda \in \sigma(A)$ there is $\lambda_{\alpha} \in$ $\sigma\left(A_{\alpha}\right)$ such that the net $\left(\lambda_{\alpha}\right)$ converges to $\lambda$, this is written

$$
\sigma(A) \subset \lim _{\alpha} \sigma\left(A_{\alpha}\right) .
$$

Proof. Let $\lambda \in \sigma(A)$ and $\varepsilon>0$ and take $\zeta=\lambda+i \varepsilon$ then:

$$
\left\|R_{\zeta}^{\alpha}\right\|_{\mathcal{L}_{\alpha}}=\frac{1}{\inf _{\rho \in \sigma\left(A_{\alpha}\right)}|\zeta-\rho|} \quad \text { and } \quad\left\|R_{\zeta}\right\|_{\mathcal{L}_{\infty}}=\frac{1}{\inf _{\rho \in \sigma(A)}|\zeta-\rho|}=\frac{1}{\varepsilon} .
$$

From the assumption, the net of resolvents strongly converges hence by Proposition 16

$$
\limsup _{\alpha} \inf _{\rho \in \sigma\left(A_{\alpha}\right)}|\zeta-\rho| \leq \varepsilon
$$

and as it is true for any $\varepsilon$, we can conclude.

Lemma 44. For any reals $a, b$ out of the spectra of $A$ such that $-\infty \leq a$ $<b \leq+\infty$ then

$$
\left.\left.a \leq \frac{\mathcal{E}(u)}{\|u\|_{\infty}^{2}} \leq b \quad \text { for every } u \in E(] a, b\right]\right) L_{\infty}^{2} \backslash\{0\} .
$$

(where $E(] a, b])=E(] a,+\infty[)$ if $b=+\infty)$.

Proof. Let $a<b$ two reals out of the spectra of $A$ and

$$
u \in E(] a, b]) L_{\infty}^{2} \backslash\{0\},
$$

then

$$
\left.\left.\int_{] a, b]} d E u=E(] a, b\right]\right) u=u=\int_{\mathbb{R}} d E u .
$$

Thus $\langle E u, u\rangle=0$ on $\mathbb{R} \backslash] a, b]$. Now if $u \in D(A)$,

$$
\mathcal{E}(u)=\langle A u, u\rangle=\int_{\mathbb{R}} \lambda d\langle E(\lambda) u, u\rangle=\int_{] a, b]} \lambda d\langle E(\lambda) u, u\rangle
$$

and the last term satisfies

$$
\begin{aligned}
a\|u\|_{\infty}^{2} & =a \int_{] a, b]} d\langle E(\lambda) u, u\rangle \\
& \leq \int_{] a, b]} \lambda d\langle E(\lambda) u, u\rangle \leq b \int_{] a, b]} d\langle E(\lambda) u, u\rangle=b\|u\|_{\infty}^{2} .
\end{aligned}
$$


For any Borel set $I \subset \mathbb{R}$ we write $n(I)=\operatorname{dim} E(I) L_{\infty}^{2}$ and $n_{\alpha}(I)=$ $\operatorname{dim} E_{\alpha}(I) L_{\alpha}^{2}$.

Proposition 45. Let $a<b$ two reals out of the point spectrum of $A$. If $\Sigma_{\alpha} \rightarrow \Sigma$ strongly then

$$
\left.\left.\left.\left.\liminf _{\alpha} n_{\alpha}(] a, b\right]\right) \geq n(] a, b\right]\right)
$$

and in particular,

$$
\liminf _{\alpha} \operatorname{dim} L_{\alpha}^{2} \geq \operatorname{dim} L_{\infty}^{2}
$$

Proof. Let us consider an orthonormal basis $\left.\left.\left\{\varphi_{k} \mid k=1, \ldots, n(] a, b\right]\right)\right\}$ of $E(] a, b]) L_{\infty}^{2}$. Let $n \in \mathbb{N}$ be a fixed number if $\left.\left.n(] a, b\right]\right)=\infty$ else $\left.\left.n=n(] a, b\right]\right)$. Then there are nets $\varphi_{k}^{\alpha} \in L_{\alpha}^{2}$ for $k=1, \ldots, n$ such that $\lim _{\alpha} \varphi_{k}^{\alpha}=\varphi_{k}$. As $\left.\left.\left.\left.E_{\alpha}(] a, b\right]\right) \rightarrow E(] a, b\right]\right)$ strongly, taking $\left.\left.\psi_{k}^{\alpha}=E_{\alpha}(] a, b\right]\right) \varphi_{k}^{\alpha}$ we get

$$
\left.\left.\lim _{\alpha} \psi_{k}^{\alpha}=E(] a, b\right]\right) \varphi_{k}=\varphi_{k}
$$

hence

$$
\lim _{\alpha}\left\langle\psi_{i}^{\alpha}, \psi_{j}^{\alpha}\right\rangle_{\alpha}=\left\langle\varphi_{i}, \varphi_{j}\right\rangle=\delta_{i j}
$$

from which we deduce that $\left(\psi_{k}^{\alpha}\right)_{k=1, \ldots, n}$ is a free family for $\alpha$ large enough and

$$
\left.\left.\lim _{\alpha} n_{\alpha}(] a, b\right]\right) \geq n .
$$

This proves the first assertion. For the second it comes from the fact that $n(] a, b])$ converges to $\operatorname{dim} L_{\infty}^{2}$ as $a \rightarrow-\infty$ and $b \rightarrow+\infty$.

Let us now have a look at the compact convergence case:

Theorem 46. If $\Sigma_{\alpha} \rightarrow \Sigma$ compactly converges, then for any $a, b$ out of the point spectrum of $A$ such that $a<b$ for $\alpha$ large enough we have $\left.\left.n_{\alpha}(] a, b\right]\right)=$ $n(] a, b])$. In particular the limit of the sets $\sigma\left(A_{\alpha}\right)$ coincides with $\sigma(A)$.

Proof. The compact convergence implies that the operators $R_{\zeta}, T_{t}$ and $E(] \lambda, \mu])$ are compact (see Proposition 16). Thus the spectrum of $A$ is discrete and $n(] a, b])<\infty$ if $a<b<\infty$. Let $(0 \leq) \lambda_{1} \leq \lambda_{2} \leq \cdots \leq \lambda_{n}$ be the spectrum of $A$, where

$$
\begin{cases}n=0 & \text { if the spectrum is empty } \\ n \in \mathbb{N} & \text { if the spectrum is finite, and } \\ n=\infty & \text { if the spectrum is a sequence converging to infinity. }\end{cases}
$$

Step 1. Fix $\varepsilon_{0}$ and let $\left.\left.\Lambda_{1}^{\alpha}=E(]-\infty, \lambda_{1}+\varepsilon_{0}\right]\right) L_{\alpha}^{2}$ and $\Lambda_{1}=L_{\infty}^{2}$, where $\lambda_{1}=\lambda_{1}+\varepsilon_{0}=\infty$ if $n=0$. Let

$$
\mu_{1}=\liminf _{\alpha} \inf \left\{\mathcal{E}_{a}(u) \mid\|u\|_{\alpha}=1, u \in \Lambda_{1}^{\alpha}\right\} .
$$

Lemma 44 allows us to say that $\left.\left.\lim _{\alpha} n_{\alpha}(]-\infty, \mu\right]\right)=0$ for any $\left.\mu \in\right]-\infty, \mu_{1}[$. Applying Proposition 45 we get $n(]-\infty, \mu])=0$, in other words for any 
$\mu \leq \mu_{1}$ then $\mu \leq \lambda_{1}$ thus $\mu_{1} \leq \lambda_{1}$. Hence if $\mu_{1}=+\infty, n=0$ and $L_{\alpha}^{2}=0$ for $\alpha$ large enough and the theorem is proved in that case.

Suppose that $\mu_{1}<+\infty$. For $\alpha$ large enough we can find unit vectors $\varphi_{1}^{\alpha} \in \Lambda_{1}^{\alpha}$ such that $\liminf _{\alpha} \mathcal{E}_{\alpha}\left(\varphi_{1}^{\alpha}\right)=\mu_{1}$. From the asymptotic compactness of $\mathcal{E}_{\alpha}$ we can extract a subnet $\left(\varphi_{1}^{\alpha}\right)_{\alpha \in \mathcal{A}}$ such that $\varphi_{1}=\lim _{\alpha} \varphi_{1}^{\alpha}$ strongly and thanks to Definition $20 \mathcal{E}\left(\varphi_{1}\right) \leq \mu_{1}$. The strong convergence induces the convergence of the norms hence $\left\|\varphi_{1}\right\|=1$ and

$$
\lambda_{1}=\inf \left\{\mathcal{E}(u) \mid\|u\|=1, u \in \Lambda_{1}\right\} \leq \mathcal{E}\left(\varphi_{1}\right) \leq \mu_{1}<+\infty .
$$

As a consequence $n \geq 1, \lambda_{1}=\mu_{1}=\mathcal{E}\left(\varphi_{1}\right)$ and $\varphi_{1}$ is eigenvector of $A$ for $\lambda_{1}$.

Furthermore let us notice that as $\left.\left.\left.\left.E_{\alpha}(] \lambda_{1}-\epsilon, \lambda_{1}+\epsilon\right]\right) \rightarrow E(] \lambda_{1}-\epsilon, \lambda_{1}+\epsilon\right]\right)$ strongly for any $\epsilon>0$ fixed and $\left.\left.E(] \lambda_{1}-\epsilon, \lambda_{1}+\epsilon\right]\right) \rightarrow E\left(\left\{\lambda_{1}\right\}\right)$ strongly when $\epsilon \rightarrow 0$ there is a net of positives numbers $\epsilon_{1}^{\alpha} \rightarrow 0$ such that $E_{\alpha}(] \lambda_{1}-$ $\left.\left.\epsilon_{1}^{\alpha}, \lambda_{1}+\epsilon_{1}^{\alpha}\right]\right) \rightarrow E\left(\left\{\lambda_{1}\right\}\right)$ strongly. From this we obtain a net

$$
\left.\left.\psi_{1}^{\alpha}=E_{\alpha}(] \lambda_{1}-\epsilon_{1}^{\alpha}, \lambda_{1}+\epsilon_{1}^{\alpha}\right]\right) \varphi_{1}^{\alpha} \rightarrow E\left(\left\{\lambda_{1}\right\}\right) \varphi_{1}=\varphi_{1} .
$$

Step 2. Let $\left.\left.\Lambda_{2}^{\alpha}=E(]-\infty, \lambda_{2}+\varepsilon_{0}\right]\right) L_{\alpha}^{2} \cap\left\langle\varphi_{1}^{\alpha}\right\rangle^{\perp}, \Lambda_{2}=\left\langle\varphi_{1}\right\rangle^{\perp}$ and

$$
\mu_{2}=\liminf _{\alpha} \inf \left\{\mathcal{E}_{a}(u) \mid\|u\|_{\alpha}=1, u \in \Lambda_{2}^{\alpha}\right\} .
$$

Again Lemma 44 allows us to say that $\left.\left.\lim _{\alpha} n_{\alpha}(]-\infty, \mu\right]\right)=0$ for any $\mu \in$ (]$\mu_{1}, \mu_{2}[)$ and Proposition 45 that $\mu_{2} \leq \lambda_{2}$. Hence if $\mu_{2}=+\infty$, we have $n=1$ and $L_{\alpha}^{2}=\left\langle\psi_{1}^{\alpha}\right\rangle$ for $\alpha$ large enough. Assume $\mu_{2}<\infty$. Take the unitary vectors $\varphi_{2}^{\alpha} \in \Lambda_{2}^{\alpha}$ such that $\liminf _{\alpha} \mathcal{E}_{\alpha}\left(\varphi_{2}^{\alpha}\right)=\mu_{2}$. Then the same discussion as Step 1 gives $n \geq 2, \lambda_{2}=\mu_{2}$ and the strong convergence of a subnet of $\left(\varphi_{2}^{\alpha}\right)$ to $\varphi_{2}$ an eigenvector of $A$ for the eigenvalue $\lambda_{2}$. We also find a net $\epsilon_{2}^{\alpha} \rightarrow 0$ such that $\left.\left.\psi_{2}^{\alpha}=E_{\alpha}(] \lambda_{2}-\epsilon_{2}^{\alpha}, \lambda_{2}+\epsilon_{2}^{\alpha}\right]\right) L_{\alpha}^{2} \rightarrow \varphi_{2}$. Now let us notice that for any $\epsilon>0$ there is $\alpha_{\epsilon} \in \mathcal{A}$ such that for all $\alpha \geq \alpha_{\epsilon}$ we have:

(1) $\left.\left.\psi_{i}^{\alpha} \in E_{\alpha}(] \lambda_{i}-\epsilon, \lambda_{i}+\epsilon\right]\right) L_{\alpha}^{2}$ for $i=1,2$;

(2) if $\lambda_{1}+2 \epsilon<\lambda_{2}$ then

$$
\left.\left.\left.\left.E_{\alpha}(] \lambda_{1}-\epsilon, \lambda_{1}+\epsilon\right]\right) L_{\alpha}^{2}=\left\langle\psi_{1}^{\alpha}\right\rangle \quad \text { and } \quad E_{\alpha}(] \lambda_{1}+\epsilon, \lambda_{2}-\epsilon\right]\right) L_{\alpha}^{2}=0 .
$$

Step 3. We repeat this procedure. Setting

$$
\left.\left.\Lambda_{k}^{\alpha}=E(]-\infty, \lambda_{k}+\varepsilon_{0}\right]\right) L_{\alpha}^{2} \cap\left\langle\psi_{1}^{\alpha}, \ldots, \psi_{k-1}^{\alpha}\right\rangle^{\perp}
$$

we have

$$
\lambda_{k}=\mu_{k}=\liminf _{\alpha} \inf \left\{\mathcal{E}_{a}(u) \mid\|u\|_{\alpha}=1, u \in \Lambda_{k}^{\alpha}\right\}
$$

for $k \leq n$. Let $k \in\{1,2, \ldots, n\}$ and $\epsilon>0$ be sufficiently small compared with $k$. Then, there exists $\alpha_{k, \epsilon} \in \mathcal{A}$ such that for any $\alpha \geq \alpha_{k, \epsilon}$ :

(1) For each $\lambda \in\left\{\lambda_{1}, \ldots, \lambda_{k-1}\right\}$ with $\lambda<\lambda_{k}$,

$$
\left.\left.E_{\alpha}(] \lambda-\epsilon, \lambda+\epsilon\right]\right) L_{\alpha}^{2}=\left\langle\psi_{i}^{\alpha} \mid p_{\lambda} \leq i \leq q_{\lambda}\right\rangle,
$$

where $p_{\lambda}=\min \left\{i \in \mathbb{N} \mid \lambda_{i}=\lambda\right\}$ and $q_{\lambda}=\max \left\{i \in \mathbb{N} \mid \lambda_{i}=\lambda\right\}$; 
(2) for each $i=1, \ldots, k-1$ with $\lambda_{i}<\lambda_{i+1}$,

$$
\left.\left.E_{\alpha}(] \lambda_{i}+\epsilon, \lambda_{i+1}-\epsilon\right]\right) L_{\alpha}^{2}=\{0\} .
$$

Conclusion. Let $a, b \in \mathbb{R}^{+} \backslash \sigma(A)$ two given real numbers such that $a<b$, then from what precedes we have for $\alpha$ large enough

$$
\left.\left.\left.E_{\alpha}(] a, b\right]\right) L_{\alpha}^{2}=\left\langle\psi_{k}^{\alpha}\right| k=1, \ldots, n \text { with } a<\lambda_{k} \leq b\right\rangle .
$$

Thus $\left.\left.n_{\alpha}(] a, b\right]\right)$ coincides with the number $k$ such that $a<\lambda_{k} \leq b$, in other words $n(] a, b])$.

The proof of Theorem 21 is the same as above, but defining the $\Lambda_{k}^{\alpha}$ with the help of the $\varphi_{k}^{\alpha}$.

Acknowledgements. I would like to thank my advisor Gérard Besson for his good advice and Y. Colin de Verdière who lead me to the $\Gamma$-convergence which happened to be the adapted tool, as I hope to convince the reader, for the macroscopical geometry.

\section{References}

[Bab91] I.K. Babenko, Volume rigidity of two-dimensional manifolds, (Russian) Mat. Zametki, 48(1) (1990), 10-14, 158; translation in Math. Notes, 48(1-2) (1990), 629-632 (1991), MR 1081887, Zbl 0717.53029.

[BLP78] A. Bensoussan, J.-L. Lions and G. Papanicolaou, Asymptotic Analysis for Periodic Structures, Studies in mathematics and its applications, North Holland, Amsterdam, 1978, MR 82h:35001, Zbl 0404.35001.

[Ber86] P.H. Berard, Spectral Geometry: Direct and Inverse Problems, Springer Verlag, Lecture Notes Math., 1207, 1986, MR 88f:58146, Zbl 0608.58001.

[Bow58] F. Bowman, Introduction to Bessel Functions, Dover, 1958, MR 20 \#4007, Zbl 0083.05602 .

[BD98] A. Braides and A. Defranceschi, Homogenization of Multiple Integrals, Oxford Science Publications, 1998, MR 2000g:49014, Zbl 0911.49010.

[Bur92] D. Burago, Periodic metrics, Adv. Soviet Math., 9, Amer. Math. Soc., Providence, 1992, 205-210, MR 93c:53029, Zbl 0762.53023.

[BI94] D. Burago and S. Ivanov, Riemannian tori without conjugate points are flat, Geom. Funct. Anal., 4(3) (1994), 259-269, MR 95h:53049, Zbl 0762.53023.

[BI95] _ On asymptotic volume of tori, Geom. Funct. Anal., 5(5) (1995), 800808, MR 96h:53041, Zbl 0846.53043.

[Cha94] I. Chavel, Riemannian Geometry: A Modern Introduction, Cambridge tracts in mathematics, 108, Cambridge University Press, 1994, MR 95j:53001, Zbl 0810.53001.

[DZ00] G. Duro and E. Zuazua, Large time behavior for convection-diffusion equations in $\mathbb{R}^{n}$ with periodic coefficients, J. Differential Equations, 167 (2000), 275-315, MR 2001j:35157, Zbl 0962.35086.

[Fed69] H. Federer, Geometric Measure Theory, Springer Verlag, 1969, MR 41 \#1976, Zbl 0176.00801. 
[Fuk90] Fukaya, Hausdorff convergence of Riemann manifolds and its application, in 'Recent Topics in Differential and Analytic Geometry', T. Ochiai, ed., Advanced Studies in Pure Mathematics, 18-I, Academic Press, Boston, 1990, 143-238, MR 92k:53076, Zbl 0754.53004.

[GHL90] S. Gallot, D. Hulin and J. Lafontaine, Riemannian Geometry, Universitext, Springer-Verlag, second edition, 1990, MR 91j:53001, Zbl 0716.53001.

[KKO97] A. Kasue, H. Kumura and Y. Ogura, Convergence of heat kernels on a compact manifold, Kyushu J. Math., 51 (1997), 453-524, MR 98e:58178, Zbl 0914.58031.

[KS00] M. Kotani and T. Sunada, Albanese maps and off diagonal long time asymptotics for the heat kernel, Commun. Math. Phys., 209 (2000), 633-670, MR 2001h:58036, Zbl 0953.58022.

[KS] K. Kuwae and T. Shioya, Convergence of spectral structures: A functional analytic theory and its applications to spectral geometry. Preprint.

[Laf74] J. Lafontaine, Sur le volume de la variété de Jacobi d'une variété riemannienne, C.R. Acad. Sc. Paris, 278 (1974), 1519-1522, MR 49 \#11447, Zbl 0288.53036.

[Mas93] Dal Maso, An Introduction to $\Gamma$-Convergence, Birkhäuser, Boston, 1993, MR 94a:49001, Zbl 0816.49001.

[Mas96] D. Massart, Normes Stables des Surfaces, Thèse de Doctorat, École Normale Superieure de Lyon, 1996.

[MS86] V.D. Milman and G. Schechtman, Asymptotic Theory of Finite Dimensional Normed Spaces, Lecture Notes Math., 1200, Springer Verlag, 1986, MR 87m:46038, Zbl 0606.46013.

[Mos94] U. Mosco, Composite media and asymptotic Dirichlet forms, J. Funct. Anal., 123(2) (1994), 368-421, MR 95d:47088, Zbl 0808.46042.

[Pan82] P. Pansu, Géométrie du Groupe de Heisenberg, Thèse de Docteur 3ème cycle, Université Paris VII, 1982.

[Pan99]__ Profil isopérimétrique, métriques périodiques et formes d'équilibre des cristaux, ESAIM Control Optim. Calc. Var., 4 (1999), 631-665 (electronic), MR 2002g:53051, Zbl 0939.53022.

[Rud91] W. Rudin, Functional Analysis, International series in Pure and Applied Mathematics, McGraw-Hill, second edition, 1991, MR 92k:46001, Zbl 0867.46001.

[Ver01] C. Vernicos, Spectres Asymptotiques des Nilvariétés Graduées, Thèse de Doctorat, Université Grenoble I, Joseph Fourier, 2001.

[ZKON79] V.V. Zhikov, S.M. Kozlov, O.A. Oleinik and Kha T'en Ngoan, Averaging and G-convergence of differential operators, Russian Math. Surveys, 34(5) (1979), 65-147, MR 82f:35032, Zbl 0445.35096.

INSTITUT DE MATHÉMATIQUE

UNIVERSITÉ DE NEUCHÂTEL

Rue Émile Argand 11

CH 2007 NEUCHÂTEL

SWITZERLAND

E-mail address: Constantin.Vernicos@unine.ch 